\title{
Entropy and finiteness of groups with acylindrical splittings
}

\author{
Filippo Cerocchi and Andrea Sambusetti
}

\begin{abstract}
We prove that there exists a positive, explicit function $F(k, E)$ such that, for any group $G$ admitting a $k$-acylindrical splitting and any generating set $S$ of $G$ with $\operatorname{Ent}(G, S)<E$, we have $|S| \leq F(k, E)$. We deduce corresponding finiteness results for classes of groups possessing acylindrical splittings and acting geometrically with bounded entropy: for instance, $D$-quasiconvex $k$-malnormal amalgamated products acting on $\delta$-hyperbolic spaces or on CAT( 0$)$-spaces with entropy bounded by $E$. A number of finiteness results for interesting families of Riemannian or metric spaces with bounded entropy and diameter also follow: Riemannian 2-orbifolds, non-geometric 3-manifolds, higher dimensional graph manifolds and cusp-decomposable manifolds, ramified coverings and, more generally, CAT(0)-groups with negatively curved splittings.
\end{abstract}

Mathematics Subject Classification (2020). Primary 20F65; Secondary 53C23, 57K30, 20E08, 20F69.

Keywords. Acylindrical splittings, entropy, Gromov hyperbolic spaces, CAT(0)-spaces, 2-dimensional orbifolds, 3-manifolds, ramified coverings, high dimensional graph manifolds.

\section{Contents}

Introduction . . . . . . . . . . . . . . . . . 756

1 Groups with acylindrical splittings . . . . . . . . . . . 765

2 Free subgroups . . . . . . . . . . . . . . . . . . 770

3 Applications . . . . . . . . . . . . . . . . . . 775

4 Examples . . . . . . . . . . . . . . . . . . 778

Appendices . . . . . . . . . . . . . . . . . . . 788

A Acylindrical splittings of hyperbolic 2-orbifolds _ . . . . . . . 788

B 3-manifolds with prescribed fundamental group . . . . . . . . 790

C Malnormal subgroups of CAT(0)-groups . . . . . . . . . . . 793

References . . . . . . . . . . . . . . . . . . . . 795 


\section{Introduction}

In this paper we are interested in finitely generated groups $G$ admitting $k$-acylindrical splittings, that is isomorphic to the fundamental group of a graph of groups such that the action of $G$ on the corresponding Bass-Serre tree is (non elementary and) $k$-acylindrical. The notion of acylindricity is due to Sela in [59], and arises naturally in the context of Bass-Serre theory. It is a geometric translation of the notion of malnormal amalgamated product, introduced by Karras and Solitar [36] at the beginning of the seventies (see $\S 1$ for details). We recall that an action without inversions of a finitely generated group on a simplicial tree $\mathcal{T}$ is said to be $k$-acylindrical if the fixed point set of any element $g \in G$ has diameter at most $k$.

The most elementary examples of groups possessing a $k$-acylindrical splitting are the free products and the fundamental groups of compact surfaces of negative Euler characteristic, but the class is considerably larger and encompasses several interesting classes of amalgamated groups which naturally arise in Riemannian and metric geometry, as we shall see. During the years, the existence of acylindrical actions on simplicial trees has been mainly used to prove some accessibility results ([59] and [34]). More recently, there has been an increasing interest on groups acting acylindrically on Gromov hyperbolic spaces (see [50], [45], [61], and references therein for this more general notion of acylindricity). We shall instead focus on growth and finiteness properties of such groups and of some classes of spaces arising as quotients of geometric actions of these groups.

Recall that the entropy, or exponential growth rate, of a group $G$ with respect to a finite generating set $S$ is defined as

$$
\operatorname{Ent}(G, S):=\lim _{n \rightarrow+\infty} \frac{1}{n} \log \left|S^{n}\right|,
$$

where $\left|S^{n}\right|$ is the cardinality of the ball of radius $n$ centered at the identity element, with respect to the word metric relative to $S$. We will also deal with groups $G$ acting discretely by isometries on general (non-compact) proper metric spaces $Y$; in this case, the entropy of the action is defined as

$$
\operatorname{Ent}(G \curvearrowright Y):=\limsup _{R \rightarrow+\infty} \frac{1}{R} \log \left|B_{Y}\left(y_{0}, R\right) \cap G y_{0}\right|,
$$

where $B_{Y}\left(y_{0}, R\right)$ denotes the ball of radius $R$ centred at $y_{0}$ (the limit is clearly independent from the choice of the base point $\left.y_{0} \in Y\right)$. Notice that when $Y$ is the Cayley graph $\mathrm{C}(G, S)$ of $(G, S)$ one has $\operatorname{Ent}(G, S)=\operatorname{Ent}(G \curvearrowright \mathcal{C}(G, S))$.

When $X$ is a closed Riemannian manifold it is customary to call (volume-)entropy of $X$ the entropy of $G=\pi_{1}(X)$ acting on its Riemannian universal covering space $Y=\tilde{X}$; this number coincides with the exponential growth rate of the volume of balls in $X$, and it is well known that it equals, in non-positive curvature, the topological entropy of the geodesic flow on the unitary tangent bundle of $X$, 
cf. [42]. We extend this terminology to any compact metric space $X$ obtained as the quotient $X=G \backslash Y$ of a simply connected, geodesic space $Y$ by a discrete group of isometries (possibly, with fixed points). We will come back shortly to the analytic information encrypted in this asymptotic invariant for Riemannian manifolds.

The first main result of this paper, from a group-theoretic point of view, is the following.

Theorem 1 (entropy-cardinality inequality). Let $G$ be a group acting by automorphisms without inversions, non-elementarily and $k$-acylindrically on a tree. For any finite generating set $S$ of $G$ we have

$$
\operatorname{Ent}(G, S) \geq \frac{1}{(200+72 k)} \log (|S|-1) .
$$

A first remarkable example of entropy-cardinality inequality was given by Arzhantseva and Lysenok [5]: for any given hyperbolic group $G$ there exists a constant $\alpha(G)$ such that for any non-elementary, finitely generated subgroup $H$ and any finite generating set $S$ of $H$ the inequality $\operatorname{Ent}(H, S) \geq \log (\alpha(G) \cdot|S|)$ holds. One interest of similar inequalities is that they generally represent a step forward to prove (or disprove) the realizability of the algebraic entropy

$$
\operatorname{Ent}_{\text {alg }}(G)=\inf \{\operatorname{Ent}(G, S) \mid S \text { finite generating set for } G\} .
$$

The entropy-cardinality inequality proved in this paper is a quantitative version of Arzhantseva-Lysenok's inequality, in a simpler framework; however, it has a different theoretical meaning, since the cardinality of $S$ is bounded in terms of a universal function, only depending on the entropy and the acylindricity constant $k$, and not on the group $G$ itself. Notice that it yields the lower bound

$$
\operatorname{Ent}_{\mathrm{alg}}(G) \geq \frac{1}{(200+72 k)} \log (r k(G)-1)
$$

for any group $G$ of rank $n \geq 3$ admitting a $k$-acylindrical action on a tree. There exist well-known lower bounds of the algebraic entropy for general amalgamated products, which, for low ranks, are sharper than the one given by (2), see [14], [42], and [16]. The meaning of our bound is that the algebraic entropy diverges as the rank becomes larger and larger; namely, the growth function of $G$ is always exponential in the rank, with base only depending on the acylindricity constant. This is clearly false for general, non-trivial (and non-elementary) nonacylindrical amalgamated products. For instance, $G=\left(\mathbb{Z}^{n} \times \mathbb{Z}_{2}\right) * \mathbb{Z}^{n}\left(\mathbb{Z}^{n} \times \mathbb{Z}_{3}\right) \cong$ $\mathbb{Z}^{n} \times\left(\mathbb{Z}_{2} * \mathbb{Z}_{3}\right)$, with the generating set $S=S_{n} \times\{a, b\}$, where $S_{n}$ generates $\mathbb{Z}^{n}$ and $a, b$ are, respectively, generators of $\mathbb{Z}_{2}$ and $\mathbb{Z}_{3}$, satisfies $\operatorname{Ent}(G, S)=$ $\operatorname{Ent}\left(\mathbb{Z}_{2} * \mathbb{Z}_{3},\{a, b\}\right)$ independently from $r k(G)=n+2$. 
The idea of proof of (1) is relatively elementary and is based on the construction of free subgroups of $G$ of large rank. Any collection of hyperbolic elements of $G$ admitting disjoint domains of attraction generates a free subgroup (namely, a Schottky subgroup) by a classical ping-pong argument. So, the strategy for Theorem 1 is to show that from any sufficiently large generating set $S$ one can produce large collections (compared to $|S|$ ) of hyperbolic elements with uniformily bounded $S$-length, all of whose axes are mutually distinct and at bounded distance (with respect to their translational lengths) from some base point $x_{0}$. The construction closely follows [5].

In the second part of the paper we focus on algebraic and geometric applications of the entropy-cardinality inequality. As an immediate consequence, we get general finiteness results for abstract groups admitting $k$-acylindrical splittings with uniformily bounded entropy, provided we know that they possess a complete set of relators of uniformily bounded length (cf. definitions in $\S 3$ and Theorem 3.1). The following are particularly interesting cases:

Corollary 2. The number of isomorphism classes of marked, $\delta$-hyperbolic groups $(G, S)$ admitting a k-acylindrical splitting and satisfying $\operatorname{Ent}(G, S) \leq E$ is finite, bounded by an explicit function $M(k, \delta, E)$.

Corollary 3. The number of groups $G$ admitting a k-acylindrical splitting, with a D-quasiconvex action on

(i) either some (proper, geodesic) $\delta$-hyperbolic space $(X, d)$,

(ii) or on some $\mathrm{CAT}(0)$-space $(X, d)$,

and satisfying $\operatorname{Ent}(G \curvearrowright X) \leq E$ is finite. Their number is bounded by a function of $k, \delta, D, E$ in case ( $i)$, and of $k, D, E$ in case (ii).

We stress the fact that, in the above corollary, the hyperbolic or CAT( 0$)$-spaces $X$ the group $G$ acts on are not supposed to be fixed.

A typical case where a group $G$ admits an action on a CAT(0)-space and a $k$-acylindrical splitting occurs when $G$ is the fundamental group of a space $X=X_{1} \sqcup_{Z} X_{2}$ which is the gluing of two locally CAT(0)-spaces $X_{i}$ along two isometric, locally convex subspaces $Z_{i} \cong Z$ (or $X=X_{0} \sqcup_{\phi}$ is obtained by identifying two such subspaces $Z_{i} \subset X_{0}$ to each other by an isometry $\phi$ ), and the resulting space $X$ is locally, negatively curved around $Z$. Namely, we will say that $X$ has a negatively curved splitting if the subspace $Z$ obtained by identifying $Z_{1}$ to $Z_{2}$ has a neighbourhood $U(Z)$ in $X$ such that $U(Z) \backslash Z$ is a locally CAT $(-\kappa)$-space, for some $\kappa>0$. The fact that $Z$ possesses such a neighborhood ensures that $\pi_{1}(Z)$ is a malnormal subgroup in each $\pi_{1}\left(X_{i}\right)$, and therefore (when the splitting is non-trivial) $\pi_{1}(X)$ has a 1-acylindrical splitting, cf. $\$ 3.2$ and Appendix $\mathrm{C}$ for details. Notice that some form of strictly negative curvature must be assumed to deduce that $\pi_{1}(Z)$ is malnormal in $\pi_{1}(X)$, and it is not sufficient 
to require that $Z$ is a locally convex subset of a locally CAT(0)-space $X$ of rank 1 . A counterexample is provided, for instance, by a 3-dimensional irreducible manifold $X$ with non trivial JSJ-splitting and one component of hyperbolic type: by [40], $X$ can be given a non-positively curved metric of rank 1 in the sense of [6], and possesses a totally geodesic, embedded torus $Z$ whose fundamental group is not malnormal in $\pi_{1}(X)$.

We then prove:

Theorem 4. The number of homotopy types of compact, locally CAT(0)-spaces $X$ admitting a non-trivial negatively curved splitting, satisfying $\operatorname{Ent}(X)<E$ and $\operatorname{diam}(X)<D$ is finite.

Corollary 5. There exist only finitely many non-diffeomorphic closed, nonpositively curved manifolds $X$ of dimension different from 4 admitting a nontrivial negatively curved splitting and satisfying $\operatorname{Ent}(X)<E$ and $\operatorname{diam}(X)<D$. In dimension 4 , the same is true up to homotopy equivalence.

It is worth noticing that Corollary 5 holds more generally for manifolds $X$ with metrics of curvature of any possible sign, provided that $X$ also admits a non-trivial, negatively curved splitting (this follows from the combination of Theorem 4 with Lemma 4.1, as in the proof of Theorem 6 for non-triangular 2-orbifolds).

The question whether a given family of Riemannian manifolds contains only a finite number of topological types has a long history: the ancestor of all finiteness results is probably Weinstein's theorem [66] on finiteness of the homotopy types of pinched, positively curved, even dimensional manifolds. A few years later, Cheeger's celebrated Finiteness Theorem appeared, for closed Riemannian manifolds with bounded sectional curvature and, respectively, lower and upper bounds on volume and diameter, see [20] and [52]. Several generalizations (see [28] and [29]) with relaxed assumptions on the curvature then followed, until, in the nineties, the attention of geometers turned to Riemannian manifolds satisfying a lower bound on the Ricci curvature, driven by Gromov's Precompactness Theorem. Substantial progresses in understanding the diffeomorphism type of Gromov-Hausdorff limits and the local structure of manifolds under lower Ricci curvature bounds were then made - by no means trying to be exhaustive by Anderson and Cheeger [3], Cheeger and Colding [21] and, more recently by Kapovich and Wilking [35] (see also Breuillard, Greene, and Tao's work [12], for a more group-theoretical approach to the generalized Margulis' Lemma under packing conditions, a macroscopic translation of a lower Ricci curvature bound).

Corollary 5 represents an attempt to get rid of lower curvature bounds, at least in non-positive curvature, replacing it only by a bound of an asymptotic invariant. Recall that, for a closed Riemannian manifold $X$, a lower bound of the Ricci curvature $\operatorname{Ricci}_{X} \geq-(n-1) K^{2}$ implies a corresponding upper bound 
of the entropy $\operatorname{Ent}(X) \leq(n-1) K$, by the classical volume-comparison theorems of Riemannian geometry. However the entropy, being an asymptotic invariant, only depends on the large-scale geometry of the universal covering $\tilde{X}$, and can be seen as an averaged version of the curvature (this can be given a precise formulation in negative curvature by integrating the Ricci curvature on the unitary tangent bundle of $X$ with respect to a suitable measure, cf. [38]). Therefore, the condition $\operatorname{Ent}(X)<E$ is much weaker than a lower bound on the Ricci curvature. To get a glimpse of the difference, remark that the class of closed, Riemannian manifolds (of dimension $n \geq 3$ ) with uniformily bounded entropy and diameter is not precompact with respect to the Gromov-Hausdorff distance (see [56], Remark 2), and neither is the family of Riemannian structures with uniformily bounded entropy and diameter on any given $n$-dimensional manifold $X$ (see [55], Example 2.29).

Notice that the entropy behaves as the inverse of length under renormalization of the metric: therefore, any non-positively curved manifold (in particular, any manifold obtained by glueing of non-positively manifolds space along locally convex subspaces) has a rescaling with large diameter and $\operatorname{Ent}(M)<1$ (and is still non-positively curved). This explains that the condition on the diameter (or on some other invariant fixing the scale) is essential for finiteness.

Some basic results about families of Riemannian metric and metric-measured spaces with uniformily bounded entropy, such as lemmas à-la-Margulis, finiteness and compactness results etc., are the object of [8]; see also [18] for an abelian version of the Margulis' lemma. Other local topological rigidity results under entropy bounds have recently appeared in [51] and [19].

Under this perspective, Corollary 5 might be compared with the well-know finiteness result for negatively curved $n$-manifolds $X$ with uniformily bounded diameter and sectional curvature $K(X) \geq-k^{2}$ (which follows from a version of the Margulis' Lemma in non-positive curvature, as stated for instance in [15], and from the aforementioned Cheeger's finiteness theorem). It is a challenging open question to know whether the conclusion of Corollary 5 extends to all closed, negatively curved manifolds with uniformily bounded entropy and diameter.

The entropy-cardinality inequality becomes a powerful tool, when applied to families of Riemannian manifolds enjoying strong topological-rigidity properties. To illustrate this fact, we present now some basic examples of application of Theorem 1 to particular classes of spaces whose groups naturally possess acylindrical splittings and presentations with an uniform bound on the acylindricity constant and on the length of relators.

\section{A. Two-dimensional orbifolds of negative orbifold characteristic. Orbifolds} were introduced by Satake [57] in the late fifties under the name of $V$-manifolds and later popularized by Thurston [65] who used them to show the existence of locally homogeneous metrics on Seifert fibered manifolds. Generally speaking, 
$n$-dimensional orbifolds are mild generalization of manifolds, whose points have neighborhoods modeled on the quotient of $\mathbb{R}^{n}$ (or on the upper half space $\mathbb{R}_{+}^{n}$ ) by the action of a finite group of transformations; we refer to $\S 4.1$ for precise definitions and isomorphisms of 2-orbifolds. In the '80s Fukaya introduced the equivariant Gromov-Hausdorff distance [26], and used it to study Riemannian orbifolds. Since then, several authors gave attention to spectral and finiteness results on Riemannian orbifolds (see for instance [11], [24], [62], [54], and [53]), possibly because of their application to string theory [1]. We show:

Theorem 6. Let $\mathcal{O}^{2}(E, D)$ be the class of Riemannian, compact, 2-orbifolds (with or without boundary) with conical singularities satisfying $\operatorname{Ent}(\mathcal{O}) \leq E$ and $\operatorname{diam}(\mathcal{O}) \leq D$. This class contains only a finite number of isomorphism types.

Notice that the analogous result for compact surfaces easily follows from basic estimates of the algebraic entropy of a surface group, together with the aforementioned Gromov's inequality

$$
\operatorname{Ent}(X) \operatorname{diam}(X) \geq \frac{1}{2} \operatorname{Ent}_{\mathrm{alg}}\left(\pi_{1}(X)\right) .
$$

Actually, it is well known that the algebraic entropy of a compact surface $X$ of genus $g$ with $h$ boundary component is bounded from below by $\log (4 g+3 h-3)$ (cf. [22]), therefore $\operatorname{Ent}(X)$ and $\operatorname{diam}(X)$ bound $g$ and $h$. The orbifold case is significantly more tricky: we use the fact that non-triangular orbifolds of negative Euler characteristic always admit a 2-acylindrical splitting (a proof of this is given in the Appendix A, Proposition A.1), so we can apply the entropy-cardinality inequality to particular, well-behaved presentations of the orbifold groups. On the other hand, triangular orbifolds do not admit such splittings, and we are forced to a direct computation, using arguments from classical small cancellation theory.

Remark. The above finiteness theorem marks a substantial difference with the analogue question in geometric group theory: in fact, the number of 2-orbifold groups $G$ admitting a generating set $S$ such that $\operatorname{Ent}(G, S) \leq E$ is not finite (at least, without any additional, uniform hyperbolicity assumption on the groups $G$ ). Actually, on any topological surface $S$ of genus $g$ with $k$ conical points of orders $p_{1}, \ldots, p_{k}$ there always exists a generating set $S$ of cardinality at most $2 g+k$, such that $\operatorname{Ent}(G, S)$ is smaller than the entropy of the free group on $S$, independently from the choice of the orders $p_{1}, \ldots, p_{k}$ (we thank R. Coulon for pointing out this fact to us).

The reason for this difference is that, on Riemannian orbifolds, any torsion element $g \in G$ has a fixed point on $\tilde{X}$, which gives rise to arbitrarily small loops increasing substantially the entropy; this does not happen for the action of $G$ on its Cayley graph $\mathcal{G}(G, S)$. Notice also that the existence of torsion elements with unbounded orders prevents $(G, S)$ to be $\delta$-hyperbolic, for any fixed $\delta$. 
B. Non-geometric 3-manifolds. A compact 3-manifold, possibly with boundary, is called non-geometric ${ }^{1}$ if its interior cannot be endowed with a complete Riemannian metric locally isometric to one of the eight 3-dimensional complete, maximal, homogeneous model geometries: $\mathbb{E}^{3}, \mathbb{S}^{3}, \mathbb{H}^{3}, \mathbb{S}^{2} \times \mathbb{R}, \mathbb{H}^{2} \times \mathbb{R}, \mathbb{H}^{2} \widetilde{\times} \mathbb{R}$, Nil and Sol. We restrict our attention to orientable manifolds, for the sake of simplicity, ${ }^{2}$ without spherical boundary components (since, clearly, punctures cannot be detected by the fundamental group in dimension 3 ).

By classical results of 3-dimensional topology and by the solution of the Geometrization Conjecture, any such manifold $X$ falls into one of the following mutually disjoint classes (as explained, for instance, in [2]):

(i) either $X$ is not prime, and is different from $P^{3} \mathrm{R} \# P^{3} \mathbb{R}$ (the only compact, non-prime manifold without spherical boundary components admitting a geometric structure, modelled on $\mathbb{S}^{2} \times \mathbb{R}$ );

(ii) or $X$ is irreducible, has a non-trivial JSJ-decomposition and is not finitely covered by a torus bundle (in which case, it would admit a Sol-geometry).

In the first case, the fundamental group of $X$ admits a 1-acylindrical splitting corresponding to the prime decomposition, while in case (ii) the JSJ-decomposition induces a (at most) 4-acylindrical splitting of $\pi_{1}(X)$, by [67] (cf. also $\S 4$ in [19], and [17] for details about the degree of acylindricity of the splitting over the abelian subgroups corresponding to the JSJ tori, according to the different types of adjacent JSJ-components).

In [19] the authors examined the local rigidity properties of Riemannian, non-geometric 3-manifolds with torsionless fundamental group, under uniformly bounded entropy and diameter. Here we consider, more generally, the class $\mathcal{M}_{\mathrm{ng}}^{\partial}(E, D)$ of compact, orientable non-geometric 3-manifolds (with possibly empty, non-spherical boundary), possibly with torsion, endowed with Riemannian metrics with entropy and diameter bounded from above by two positive constants $E$ and $D$. Acylindricity of the splitting of their fundamental groups is the key to the following:

Theorem 7. The number of isomorphism classes of fundamental groups of manifolds in $\mathcal{M}_{\mathrm{ng}}^{\partial}(E, D)$ is less than $2^{2^{(1+488 E D)^{3}}}$.

Moreover, the homotopy type (and, in turns, the diffeomorphism type) of compact 3-manifolds without spherical boundary components is determined by their fundamental group up to a finite number of choices, by Johannson and Swarup

${ }^{1}$ We use here the term "geometric" as in the original definition given in [64]; in the case of manifolds with boundary, variations on this definition are possible and suitable for other purposes (i.e. uniqueness of the model geometries on each piece), see for instance [10].

${ }^{2}$ The geometrization conjecture for non-orientable 3-manifolds with boundary being not yet clearly established, as far as the authors know. 
works [31] and [63] (see the discussion in $\$ 4.2$ for details, and Theorem B.1 in Appendix B). We therefore obtain:

Corollary 8. The number of diffeomorphism types in $\mathcal{M}_{\mathrm{ng}}^{\partial}(E, D)$ is finite.

Notice that, while Theorem 7 gives an explicit (albeit ridiculously huge) estimate of the number of groups in $\mathcal{M}_{\mathrm{ng}}^{\partial}(E, D)$, Corollary 8 does not provide any explicit estimate of the number of diffeomorphism types.

Remark. The bound we found in Theorem 7 is explicit, but certainly not optimal. Once finiteness for this class is known, one might try to use more efficient, computer-assisted algorithms to find reasonable estimates of their number.

C. Ramified coverings of hyperbolic manifolds. Another interesting class of spaces whose fundamental groups admit acylindrical splittings is the one of cyclic ramified coverings of hyperbolic manifolds. The construction is due to Gromov and Thurston [27] and represents an important source of examples of manifolds admitting pinched, negatively curved metrics but not hyperbolic ones. A degree $k$ ramified cover $X_{k}$ of a hyperbolic manifold $X$ is obtained by excising a totally geodesic hypersurface with boundary $Z$ in $X$, and then glueing several copies of $X-Z$ along a " $k$-paged open book," whose leaves are copies $Z_{i}$ of $Z$ joined together at the ramification locus $R=\partial Z$. Any such covering admits a singular, locally CAT(-1) metric, and its fundamental group splits as a free product of CAT $(-1)$ groups amalgamated over the fundamental group of the locally convex subspace $Z_{1} \cup Z_{k}$ of $X_{k}$ (given by two pages of the book). We will recall in $\S 4.3$ this construction in more detail, and show that these manifolds naturally fall in the class of spaces with non-trivial negatively curved splittings. However, we will be interested in metrics with curvature of any possible sign on such manifolds. Namely, let $\mathcal{R}^{4}(E, D)$ be the space of all 4-dimensional Riemannian, cyclic ramified coverings of compact, orientable hyperbolic manifolds, whose entropy and diameter are respectively bounded by $E$ and $D$; and let $\mathcal{R}^{\neq 4}(E, D)$ the corresponding space of ramified coverings of dimensions $n \geq 2, n \neq 4$. Then:

Corollary 9. The class $\mathcal{R}^{4}(E, D)$ contains only finitely many different homotopy types, and $\mathcal{R}^{\neq 4}(E, D)$ only finitely many diffeomorphism types.

We stress the fact that the manifolds under consideration are (genuine) ramified coverings of any possible hyperbolic manifold, and not just of one fixed hyperbolic manifold.

D. High dimensional graph and cusp decomposable manifolds. High dimensional graph manifolds have been introduced by Frigerio, Lafont and Sisto in [25], and cusp-decomposable manifolds by Nguyen Phan in [49]. Roughly speaking, 
an (extended) $n$-dimensional graph manifold $X$ is obtained gluing together, via affine diffeomorphisms of their boundaries, several elementary, building blocks $X_{i}$ which are diffeomorphic to products $H^{k_{i}} \times T^{n-k_{i}}$, where $T^{n-k_{i}}$ is a $\left(n-k_{i}\right)$-dimensional torus (representing the local fibers of the graph manifold), and $H^{k_{i}}$ is a manifold of dimension $k_{i} \geq 2$ with toroidal boundary components, obtained from a hyperbolic manifold with cusps by truncating some cusps along (the quotient of) flat horospheres; a block of the form $H^{2} \times T^{n-2}$ is also called a surface piece. A high dimensional graph manifold $X$ is called irreducible if none of the fibers of two adjacent blocks represent the same element in $\pi_{1}(X)$, and purely hyperbolic if there are no fibers at all (that is every piece $X_{i}$ is a truncated $n$-dimensional hyperbolic manifold). Notice that the above class does no cover the case of nongeometric 3-manifolds, since surface pieces are trivial products, so the blocks do not take into account Seifert fibrations.

Cusp decomposable manifolds are defined similarly to purely hyperbolic high dimensional graph manifolds, but starting from building blocks which are negatively curved, locally symmetric manifolds with cusps, and glueing, always via affine diffeomorphisms, ${ }^{3}$ the boundary infra-nilmanifolds obtained by truncating the cusps. We say that a $n$-dimensional graph manifold, or a cusp decomposable manifold $X$, is non-elementary if it is obtained by identifying at least two boundaries (of one or more building blocks).

High dimensional graph enjoy strong topological rigidity properties, as they are aspherical spaces and satisfy the Borel Conjecture in dimension $n \geq 6$ (cf. [25], Theorem 3.1 and \$3.4, Remark 3.7); moreover, the diffeomorphism type of (closed) cusp decomposable manifolds, or of all high dimensional graph manifolds whose boundary components do not belong to surface pieces, is determined by the fundamental group within the respective classes (cf. Theorem 0.7 in [25] and [49]). The importance of considering affine gluings, in order to get rigidity, was pointed out by Aravinda and Farrell in [4], where they considered the so called twisted doubles - which also are included in the class defined in [25].

Non-elementary, irreducible high dimensional graph manifolds groups admit 2-acylindrical splittings, while cusp decomposable manifolds (or purely hyperbolic, high dimensional graph manifolds) groups have 1-acylindrical splittings. Therefore, we obtain:

\footnotetext{
${ }^{3}$ An affine diffeomorphism, in this context, is the composition of an isomorphism of the two nilpotent Lie groups composed with the left multiplication by an element of the group. In [49] the author points out the necessity of realizing the gluings via affine diffeomorphisms to have strong differential rigidity: in fact, Aravinda and Farrell show in [4] the existence of non-affine gluing maps, for the double of a hyperbolic cusped manifold $X$, giving rise to a manifold which is not diffeomorphic to the one obtained by gluing the two copies via the identity map of $\partial X$.
} 
Corollary 10. Let $\mathcal{G}^{\partial}(E, D)$ (resp. $\mathcal{G}(E, D)$ ) be the class of compact (resp. closed) Riemannian, non-elementary irreducible high dimensional graph manifolds with entropy and diameter bounded by $E, D$, and let $\mathcal{C}(E, D)$ be the class of closed Riemannian, non-elementary cusp decomposable manifolds satisfying the same bounds:

(i) $\mathcal{G}^{\partial}(E, D)$ contains finitely many homotopy types, and $\mathcal{G}(E, D)$ only finitely many diffeomorphism types;

(ii) $\mathcal{C}(E, D)$ contains a finite number of diffeomorphism types.

Notice that we do not bound a priori the dimension of the manifolds in these classes: this follows, in the aftermath, from bounding their entropy and diameter.

Moreover, notice that, while the results on Riemannian 2-orbifolds and ramified coverings (though concerning metrics of any possible sign) still pertain to the framework of spaces of negative curvature, the classes of non-geometric 3-manifolds and of high dimensional graph or cusp decomposable manifolds escape from the realm of non-positive curvature. This is clear for non-prime 3-manifolds, and follows from Leeb's work [40] for irreducible 3-manifolds. Cusp decomposable manifolds obtained by gluing boundary infra-nilmanifolds which are not tori do not admit nonpositively curved metrics, by the Solvable Subgroup Theorem; also, in [25] there are examples of $n$-dimensional graph manifolds not supporting any locally CAT(0)-metric, for $n \geq 4$.

The paper is structured as follows. In $\S 1$ we recall some basic facts about acylindrical actions on trees and acylindrical splittings of groups, recalling the relation with the notions of malnormal amalgamated products and HNN-extensions. $\$ 2$ is devoted to the proof of the entropy-cardinality inequality. As a consequence of this inequality, in $\S 3$ we derive some general, finiteness results for groups admitting acylindrical splittings.

Finally, $\S 4$ is devoted to describing a number of geometrically interesting families of spaces admitting such splittings: 2-dimensional orbifolds, non-geometric 3-manifolds, high-dimensional graph manifolds, cusp decomposable manifolds and ramified coverings of hyperbolic manifolds.

Acknowledgements. The first author acknowledges financial support by the Max-Planck Institut für Mathematik and praises the excellent working conditions provided by the Institut. Both the authors wish to thank R. Coulon for useful discussions during his stay in Rome in 2016, and S. Gallot for many precious hints. We also thank the referees for their attentive review and pertinent remarks.

\section{Groups with acylindrical splittings}

We briefly recall here some basic notions about acylindrical actions on trees. 
In the following, $\mathcal{T}$ will always denote a simplicial tree, endowed with its simplicial metric $d_{\mathcal{T}}$ (i.e. the metric which makes any edge isometric to the unit interval). Recall that, for $x, y, z \in \mathcal{T}$, the Gromov product of $y, z$ based at $x$ is defined as

$$
(y, z)_{x}=\frac{1}{2}\left(d_{\mathcal{T}}(x, y)+d_{\mathcal{T}}(x, z)-d_{\mathcal{T}}(y, z)\right)
$$

and equals the length of the common subsegment of the geodesics $[x, y]$ and $[x, z]$.

An action of a group $G$ on a simplicial tree $\mathcal{T}$ is said to be without (edge) inversions if for any oriented edge e and for any $g \in G$ we have $g e \neq \bar{e}$, where è is the edge e with the opposite orientation. In what follows, we shall always assume that the action of $G$ on $\mathcal{T}$ is by automorphisms and without inversions.

The elements of $G$ can then be divided into two classes, according to their action: elliptic and hyperbolic elements. They are distinguished by their translation length, which is defined, for $g \in G$, as

$$
\tau(g)=\inf _{\mathrm{v} \in \mathcal{T}} d_{\mathcal{T}}(\mathrm{v}, g \cdot \mathrm{v}) .
$$

If $\tau(g)=0$ the element $g$ is called elliptic, otherwise it is called hyperbolic. We will denote by $\mathrm{T}(g)$ the characteristic subset of $g$, that is the subset of $\mathcal{T}$ where $d(x, g \cdot x)=\tau(g)$. If $g$ is elliptic, then $\mathrm{T}(g)=\mathrm{Fix}(g)$, the subset of fixed points of $g$. If $g$ is hyperbolic, then $\mathrm{T}(g)$ is a bi-infinite geodesic, the axis of $g$, denoted $\operatorname{Ax}(g)$ : each element on the axis is translated of $\tau(g)$ along the axis, whereas elements at distance $d$ from the axis are translated of $\tau(h)+2 d$. We will denote by $g^{ \pm}$the points at infinity of $\operatorname{Ax}(g)$ which are, respectively, the repelling and attractive points of $g$.

To produce hyperbolic elements with controlled translation length we will need later the following well-known facts.

Lemma 1.1. Let $G$ act by automorphisms on a simplicial tree $\mathcal{T}$.

(i) If $g \in G$ satisfies $d_{\mathcal{T}}\left(x, g^{2} \cdot x\right)>d_{\mathcal{T}}(x, g x)$ then $g$ is hyperbolic, with translation length $\tau(g) \geq d_{\mathcal{T}}\left(x, g^{2} \cdot x\right)-d_{\mathcal{T}}(x, g x)$ and axis $\operatorname{Ax}(g)=$ $\bigcup_{n \in \mathbb{Z}} g^{n} \cdot[m, g \cdot m]$, where $m$ is the midpoint of the segment $[x, g x]$;

(ii) if $g \in G$ sends a couple of points $\left(v_{0}, v_{1}\right)$ in $\left(w_{0}, w_{1}\right)$, with $\left\{v_{0}, v_{1}, w_{0}, w_{1}\right\}$ collinear and of cardinality $\geq 3$, and if $d_{\mathcal{T}}\left(v_{0}, w_{0}\right)=d_{\mathcal{T}}\left(v_{1}, w_{1}\right)$ then $g$ is hyperbolic, these points belong to $\operatorname{Ax}(g)$ and $\tau(h)=d\left(v_{0}, w_{0}\right)$.

The first assertion follows from the inequality

$$
\tau(g) \geq d\left(x, g^{2} \cdot x\right)-d(x, g \cdot x)-2 \delta,
$$

which holds on every Gromov $\delta$-hyperbolic space, cf. [5] and [8]; the second one is Lemma 6 in [16], and is a reformulation of Proposition 25 in [60]. 
Definition 1.2 (acylindrical actions on trees). Let $G$ be a discrete group acting by automorphisms without edge inversions on a simplicial tree $\mathcal{T}$, endowed with its natural simplicial distance $d_{\mathcal{T}}$. The action $G \curvearrowright \mathcal{T}$ is $k$-acylindrical if the set of fixed points of every elliptic element $g \in G$ has diameter less than or equal to $k$, and that the action is acylindrical if it is $k$-acylindrical for some $k$.

The next lemma resumes some facts about the stabilizer of the axis of a hyperbolic element, which we will use in the following. For any hyperbolic $h \in G$, we call $N(h)$ the stabilizer in $G$ of the axis of $h$; we also define the oriented stabilizer of $h$ as the subset $N^{+}(h)<N(h)$ of elements $g$ fixing $h^{-}$and $h^{+}$(that is, preserving the orientation of $\operatorname{Ax}(h)$ induced by the translations by $h)$.

Lemma 1.3. Let $G \curvearrowright \mathcal{T}$ be a k-acylindrical action on a tree. Let $h \in G$ be hyperbolic, let $h_{0}$ be a hyperbolic element with $\operatorname{Ax}\left(h_{0}\right)=\operatorname{Ax}(h)$ and $\tau\left(h_{0}\right)$ minimal, and let $g \in G$.

(i) If $g$ is hyperbolic with $\operatorname{Ax}(g)=\operatorname{Ax}(h)$, then $g$ is a power of $h_{0}$;

(ii) if $g \mathrm{Ax}(h)=\operatorname{Ax}(h)$ then either $g h g^{-1}=h$ or $g h g^{-1}=h^{-1}$, depending on whether $g$ preserves the orientation of $\operatorname{Ax}(h)$ or not;

(iii) the oriented stabilizer $N^{+}(h)$ is the infinite cyclic subgroup generated by $h_{0}$ or $h_{0}^{-1}$;

(iv) the stabilizer $N(h)$ is either equal to $N^{+}(h)$ or to the infinite dihedral group $N^{+}\left(h_{0}\right) \rtimes \mathbb{Z}_{2}$ generated by $\left\{h_{0}, \sigma_{0}\right\}$, where $\sigma_{0}$ is an elliptic element of order 2 swapping $\mathrm{Ax}\left(h_{0}\right)$.

Proof. Since $h_{0}$ has minimum translation length among hyperbolic elements with the same axis, any hyperbolic $g$ with $\operatorname{Ax}(g)=\operatorname{Ax}(h)$ acts on $\operatorname{Ax}\left(h_{0}\right)$ as $h_{0}^{n}$, for some $n \in \mathbb{Z}$; hence $g=h_{0}^{n}$ necessarily, by acylindricity, which shows (i).

To see (ii), notice that if $\operatorname{Ax}(h)=g \operatorname{Ax}(h)=\operatorname{Ax}\left(g h g^{-1}\right)$ then $g h g^{-1}=h^{ \pm 1}$ by (i), since $\tau\left(g h g^{-1}\right)=\tau(h)$. Moreover, as $g$ stabilizes $\mathrm{Ax}(h)$, if $g$ is hyperbolic then it has the same axis as $h$, preserves the orientation of the axis and commutes with $h$; that is $g h g^{-1}=h$. On the other hand, if $g$ is elliptic, by acylindricity it can only swap $\operatorname{Ax}(h)$ by a reflection through a vertex (because $G$ acts without edge inversions), so $g h g^{-1}$ acts on $\mathrm{Ax}(h)$ as $h^{-1}$; this implies that $g h g^{-1}=h^{-1}$, again by acylindricity. Assertion (iii) immediately follows from (ii).

To see (iv), let $g \in N(h)$. If $g$ is hyperbolic, we know by (ii) that it belongs to the infinite cyclic subgroup $\left\langle h_{0}\right\rangle$. If $g$ is elliptic, we have seen that it acts on $\operatorname{Ax}(h)$ by a reflection through a vertex $\mathrm{v}$, so $g^{2}=1$ and $g h_{0} g^{-1}=h_{0}^{-1}$ by acylindricity. If $g^{\prime}$ is another element of $N(h) \backslash N^{+}(h)$, then also $g^{\prime}$ acts by swapping $\operatorname{Ax}(h)$ and fixing some $\mathrm{v}^{\prime} \in \operatorname{Ax}(h)$. Then, $g^{\prime} g^{-1}=g^{\prime} g$ acts on $\operatorname{Ax}(h)$ as $h_{0}^{n}$, and by acylindricity $g^{\prime} g=h_{0}^{n}$. This shows that, if $N(h) \neq N^{+}(h)$ then $N(h) \cong N^{+}\left(h_{0}\right) \rtimes \mathbb{Z}_{2}$ and is generated by $h_{0}$ and by the elliptic element $\sigma_{0}:=g$ of order 2 . 
This last lemma bounds the size of the intersection of the axes of two hyperbolic elements in terms of the acylindricity constant $k$ and of the translation lengths.

Lemma 1.4. Let $G \curvearrowright \mathcal{T}$ be a non-elementary, $k$-acylindrical action on a tree. Let $h_{1}, h_{2} \in G$ be two hyperbolic elements with distinct axes. Then,

$$
\operatorname{diam}\left(\operatorname{Ax}\left(h_{1}\right) \cap \operatorname{Ax}\left(h_{2}\right)\right) \leq \tau\left(h_{1}\right)+\tau\left(h_{2}\right)+k .
$$

Proof. We argue by contradiction. Let $I=\operatorname{Ax}\left(h_{1}\right) \cap \operatorname{Ax}\left(h_{2}\right)$ and assume that $\operatorname{diam}(I) \geq \tau\left(h_{1}\right)+\tau\left(h_{2}\right)+k+1$. Up to taking inverses, we may assume that the elements $h_{1}, h_{2}$ translate $I$ in the same direction, and we let $I$ be oriented by this direction. Then, the element $\left[h_{1}, h_{2}\right]$ would fix pointwise the initial subsegment of length $k+1$ of $I$ and this, by $k$-acylindricity, implies that $\left[h_{1}, h_{2}\right]=1$. This contradicts the assumption that $h_{1}$ and $h_{2}$ have distinct axes.

Let $\mathcal{T}_{G}$ be the minimal subtree of $\mathcal{T}$ which is $G$-invariant: the action of $G$ is said elliptic (or trivial) it $\mathcal{T}_{G}$ is a point, and linear if $\mathcal{T}_{G}$ a line; in both cases we shall say that the action of $G$ is elementary. This is equivalent to asking that the limit set $L G$ in the visual boundary $\mathcal{T}(\infty)$ of the tree is, respectively, empty or reduced to two points. Notice that an elliptic action of $G$ does not give any information on the group $G$; on the other hand, by Lemma 1.3(iv), if the action of $G$ on $\mathcal{T}$ is linear and acylindrical, then a $G$ is virtually cyclic.

Definition 1.5 (acylindrical splittings). We will say that a finitely generated group $G$ admits a k-acylindrical splitting if $G$ admits a non-elementary $k$-acylindrical action on a simplicial tree.

The main source of examples of acylindrical splittings comes from Bass-Serre theory. Actually, the notion of $k$-acylindrical action of a discrete group on a tree is a geometric reformulation which generalizes to graph of groups the notion of $k$-step malnormal amalgamated product, due to A. Karass and D. Solitar [36].

We recall that an element $g$ belonging to an amalgamated product $G=A *_{C} B$ is written in normal form when it is expressed as $g=g_{0} g_{1} g_{2} \cdots g_{n}$, where $g_{0} \in C$ and, for $i \geq 1$, no $g_{i}$ belongs to $C$ and two successive $g_{i}$ belong to different factors of the product; the integer $n=\ell(g)$ is then called the syllable length of $g$ (then, the identity and the elements of $C$ have zero syllable length by definition).

An amalgamated product $G=A *_{C} B$ is called $k$-step malnormal if one has $g C g^{-1} \cap C=\{1\}$ for all $g \in G$ with $\ell(g) \geq k+1$ (in particular, $k=0$ if and only if $C$ is a malnormal subgroup of $G$; and, by definition, free products are (-1)-malnormal). A similar definition can be given for a group $A *_{\varphi}=\left\langle A, t \mid \operatorname{rel}(A), t^{-1} c t=\varphi(c)\right\rangle$ which is a $\mathrm{HNN}$-extension of $A$ with respect to an isomorphism $\varphi: C_{-} \rightarrow C_{+}$between subgroups $C_{-}, C_{+}$. Namely, by Britton's 
Lemma every element $g \in G^{*}$ can be written in a normal form as

$$
g=g_{0} t^{\varepsilon_{1}} g_{1} \cdots g_{m-1} t^{\varepsilon_{m}} g_{m},
$$

where $g_{0} \in A, \varepsilon_{i}= \pm 1$, and $g_{i} \in A \backslash C_{\varepsilon_{i}}$ if $\varepsilon_{i+1}=-\varepsilon_{i}$; the syllable length of $g$ is defined in this case as $\ell(g)=m$. A HNN-extension $G=A *_{\varphi}$ is called $k$-step malnormal if $g C_{\varepsilon} g^{-1} \cap C_{\varepsilon^{\prime}}=\{1\}$ for any $\varepsilon, \varepsilon^{\prime} \in\{ \pm\}$ and $^{4}$ for all $g \in G$ with $\ell(g) \geq k+2$.

We will simply say that $G$ is a malnormal amalgamated product or $H N N$-extension if it is $k$-step malnormal for some $k$. Then, it is easy to check that if a group $G$ is a $(k-1)$-step malnormal amalgamated product or $\mathrm{HNN}$-extension, then the action on the Bass-Serre tree $\mathcal{G}$ of the splitting is $k$-acylindrical. Conversely, if $G$ admits a $k$-acylindrical action on a simplicial tree having a segment (resp. a loop) as underlying graph $\mathcal{G}$, then $G$ is a $(k-1)$-step malnormal amalgamated product (resp. HNN-extension); see [17] for further details.

When $G$ is a non-trivial amalgamated product $A *_{C} B$ (that is, $A \subsetneq G$ and $B \subsetneq G$ ) or a HNN extension, then $G$ always acts on the Bass-Serre tree of the splitting without global fixed points; ${ }^{5}$ hence, the action is non-elementary if and only if it is not linear. Therefore, if $G=A *_{C} B$ is a non-trivial, malnormal amalgamated product with $C$ of index greater than 2 in $A$ or $B$ (i.e. $G$ not virtually cyclic), then $G$ admits an acylindrical splitting. Analogously, if $G=A *_{\varphi}$ is a malnormal HNN extension with $C_{-} \subsetneq A$ or $C_{+} \subsetneq A$, then $G$ admits an acylindrical splitting.

In our examples, we will make use of the following facts about amalgamated products and HNN-extensions over malnormal subgroups (the proof of Lemma 1.6(i)-(ii) can be found, with the original terminology, in $\$ 5$ of [36]; the case of HNN-extensions is analogous, cf. [17] for more details). We say that two subgroups $C_{-}, C_{+}$of $G$ are conjugately separated when $g C_{-} g^{-1} \cap C_{+}$is trivial for all $g \in G$.

Lemma 1.6. Let $A *_{C} B$ be the amalgamated product of two groups $A, B$. Assume that $C$ is a proper subgroup having index greater than 2 in $A$ or in $B$, and let $\iota_{A}: C \rightarrow A$ and $\iota_{B}: C \rightarrow B$ be the natural inclusions:

(i) if one between $\iota_{A}(C), \iota_{B}(C)$ is malnormal in the respective group, then $A *_{C} B$ admits a 2-acylindrical splitting;

${ }^{4}$ This seems to contrast with the definition for amalgamated products, but it actually yields that $G=A *_{\varphi}$ is a 0 -malnormal $\mathrm{HNN}$ extension if and only if $C_{+}, C_{-}$are malnormal and conjugately separated in $A$, and that $G$ is $(-1)$-malnormal if and only if $C_{ \pm}=\{1\}$. This is due to the relation $t^{-1} C_{-} t=C^{+}$; one might express the same condition by imposing $g C_{\varepsilon} g^{-1} \cap C_{\varepsilon^{\prime}}=\{1\}$ for all $g$ with $\ell(g)=k+1$ and whose normal form satisfies some additional (awkward to write) restrictions.

${ }^{5}$ On the other hand, the fundamental group $G$ of a graph of groups can fix a point of the boundary $\mathcal{T}(\infty)$ of its Bass-Serre tree (in which case the action is quasi-parabolic, according to Gromov's classification of actions on hyperbolic spaces): this happens precisely when $G$ is a nontrivial ascending HNN extension, cf. [45]. 
(ii) if both $\iota_{A}(C)$ and $\iota_{B}(C)$ are malnormal in the respective groups, then $A *_{C} B$ admits a 1-acylindrical splitting.

Lemma 1.7. Let $A *_{\varphi}=\left\langle A, t \mid t^{-1} c t=\varphi(c)\right\rangle$ be the HNN-extension of $A$ with respect to an isomorphism of subgroups $\varphi: C_{-} \rightarrow C_{+}$: if the subgroups $C_{+}, C_{-}$ are malnormal and conjugately separated in $A$, and one of them has index at least two in $A$, then $A *_{\varphi}$ admits a 1-acylindrical splitting.

\section{Free subgroups}

Recall that a subgroup $\left\langle g_{1}, \ldots, g_{n}\right\rangle$ of $G$ generated by $n$ elements $g_{1}, \ldots, g_{n}$ is a Schottky subgroup if it is possible to find subsets $X_{i} \subset \mathcal{T}$, for $i=1, \ldots, n$, such that

(i) $\bigcup_{i=1}^{n} X_{i} \subsetneq \mathcal{T}$;

(ii) $X_{i} \cap X_{j}=\varnothing$ for $i \neq j$;

(iii) $g_{i}^{k}\left(\mathcal{T} \backslash X_{i}\right) \subseteq X_{i}$ for all $i$ and $k \neq 0$.

The $X_{i}$ 's are called (joint) domains of attraction of the $g_{i}$ 's. Any Schottky subgroup on $n$ elements is a free product of cyclic groups and has rank $n$, by a classic ping-pong argument: chosen $x_{0} \in \mathcal{T} \backslash \bigcup_{i} X_{i}$, every element represented by a reduced word $w$ on the $g_{i}^{\prime} s$ or their inverses sends $x_{0}$ into some $X_{i}$, hence it is not trivial.

To produce Schottky subgroups of large rank we will use the following lemma.

Lemma 2.1. Let $G$ be a group acting on a simplicial tree $\mathcal{T}$, and let $x_{0} \in \mathcal{T}$. Assume that $h_{1}, \ldots, h_{n}$ are hyperbolic elements with $d\left(x_{0}, A x\left(h_{i}\right)\right) \leq D$ for all $i$, translation lengths $\tau\left(h_{i}\right) \geq \tau$ and such that $\operatorname{diam}\left(A x\left(h_{i}\right) \cap A x\left(h_{j}\right)\right) \leq \Delta$ for $i \neq j$. Then, the group $\left\langle h_{1}^{p}, \ldots, h_{n}^{p}\right\rangle$ is a free, Schottky subgroup of $G$ of rank $n$, for all $p>2(D+\Delta) / \tau$.

Proof. Let us describe the domains $X_{i}$ which make $\left\langle h_{1}, \ldots, h_{n}\right\rangle$ a Schottky subgroup. Let $D_{i}\left(x_{0}\right)$ be the Dirichlet domain of $\left\langle h_{i}^{p}\right\rangle$ centered at $x_{0}$, that is

$$
D_{i}\left(x_{0}\right)=\left\{x \in \mathcal{T} \mid d_{\mathcal{T}}\left(x, x_{0}\right) \leq d_{\mathcal{T}}\left(x, h_{i}^{ \pm p} \cdot x_{0}\right)\right\}
$$

and let $X_{i}=\mathcal{T} \backslash D_{i}\left(x_{0}\right)$. It is clear that $x_{0} \in \mathcal{T} \backslash \bigcup_{i} X_{i}$ and that for all $k \neq 0$ we have $h_{i}^{k p}\left(\mathcal{T} \backslash X_{i}\right)=h_{i}^{k p}\left(D_{i}\left(x_{0}\right)\right) \subset X_{i}$.

To show (ii), let $p_{i}$ the projections of $x_{0}$ on $\operatorname{Ax}\left(h_{i}\right)$, and let $I_{i j}$ be the bridge between the axes $\operatorname{Ax}\left(h_{i}\right), \operatorname{Ax}\left(h_{j}\right)$ : that is, $I_{i j}$ is the geodesic segment joining the axes $\operatorname{Ax}\left(h_{i}\right)$ and $\operatorname{Ax}\left(h_{j}\right)$ if these axes do not intersect, while $I_{i j}:=\operatorname{Ax}\left(h_{i}\right) \cap \operatorname{Ax}\left(h_{j}\right)$ otherwise. 
Assume first that $\operatorname{Ax}\left(h_{i}\right) \cap \operatorname{Ax}\left(h_{j}\right)=\emptyset$ and that the geodesic joining $x_{0}$ to $I_{i j}$ does not intersect the axes: then, it is clear that $X_{i} \cap X_{j}=\emptyset$ (as they belong to different connected components of $\mathcal{T} \backslash\left\{x_{i j}\right\}$, where $x_{i j}$ is the projection of $x_{0}$ to $I_{i j}$ ).

Otherwise, (up to switching $i$ with $j$ ) we may assume that $p_{i} \in\left[x_{0}, p_{j}\right]$. As all the axes stay at distance at most $D$ from $x_{0}$, and their (possibly empty) intersections have diameter less than $\Delta$, we deduce that $d\left(x_{0}, x\right) \leq D+\Delta$ for all $x \in I_{i j}$. Then,

$$
d\left(x, x_{0}\right)<\frac{1}{2} d\left(x, h^{p} \cdot x_{0}\right)=\frac{1}{2} p \tau
$$

for every $x \in I_{i j}$, that is $I_{i j} \subset D_{i}\left(x_{0}\right)$. This implies that the projection of $X_{j}$ on $\operatorname{Ax}\left(h_{i}\right)$ is included in $D_{i}\left(x_{0}\right)$, while obviously $X_{i}$ projects outside it. Therefore $X_{i}$ and $X_{j}$ have disjoint projections on $\operatorname{Ax}\left(h_{i}\right)$, which proves (ii).

Then, to prove Theorem 1, we will produce a large number of hyperbolic elements $g_{i} \in G$, with controlled word length $\left|g_{i}\right|_{S}$, all of whose axes stay at bounded distance in $\mathcal{T}$ from some fixed point $x_{0}$. We will denote by $S^{n}$ the subset of $G$ made of elements with $|g|_{S} \leq n$. The first step is the following lemma.

Lemma 2.2. Let $G$ be a group acting $k$-acylindrically without inversions on a simplicial tree $\mathcal{T}$, and let $S$ any finite generating set for $G$. Then, there exist $x_{0} \in \mathcal{T}$ and a hyperbolic element $g_{0} \in G$ such that

(i) $\left|g_{0}\right|_{S} \leq 33+12 k$;

(ii) $\tau\left(g_{0}\right) \geq 7+4 k$;

(iii) $d_{\mathcal{T}}\left(x_{0}, s \cdot x_{0}\right) \leq \frac{1}{7+6 k}\left(\tau\left(g_{0}\right)+2 k\right)$ for all $s \in S$;

(iv) $d_{\mathcal{T}}\left(x_{0}, \operatorname{Ax}\left(g_{0}\right)\right) \leq \frac{1}{7+6 k}\left(3 \tau\left(g_{0}\right)+13 k+6 k^{2}\right)$;

(v) $N^{+}\left(g_{0}\right)=\left\langle g_{0}\right\rangle$.

Proof. The proof closely follows [5], with minor modifications due to our simpler context. Consider the minimal joint displacement of elements of $S$

$$
L:=\inf _{x \in \mathcal{T}} \max _{s \in S} d_{\mathcal{T}}(x, s \cdot x) .
$$

Since $\mathcal{T}$ is a tree, the infimum is achieved at some $x_{0}$. In particular, $L \geq 1$ or $G$ would fix a point, which is excluded since the action is assumed non-elementary.

We will first find some $h \in G$ with $|h|_{S} \leq 2, \tau(h) \in[L, 2 L]$ and $x_{0} \in \operatorname{Ax}(h)$. In fact, either $S \cup S^{-1}$ contains a hyperbolic element $s$ such that $\tau(s)=L$, and setting $h=s$ we are done, or we have $\tau(s)<L$ for every $s \in S \cup S^{-1}$. In this case, let $s_{0} \in S$ be an element realizing the minimal joint displacement. We claim that we can find another $s_{1} \in S \cup S^{-1}, s_{1} \neq s_{0}$, with $d_{\mathcal{T}}\left(x_{0}, s_{1} \cdot x_{0}\right)=L$ too, and such that the geodesic segment from $s_{0} \cdot x_{0}$ to $s_{1} \cdot x_{0}$ contains $x_{0}$ (that is, 
$\left.\left(s_{0} \cdot x_{0}, s_{1} \cdot x_{0}\right)_{x_{0}}=0\right)$. Actually, let $S_{0}$ be the subset of elements $s \in S \cup S^{-1}$ satisfying $d\left(x_{0}, s \cdot x_{0}\right)=L$, and assume that Choose some positive $\epsilon$ such that $\epsilon<\frac{1}{2} \min \left\{\varepsilon_{0}, L-\max _{s \in S \backslash S_{0}} d\left(x_{0}, s \cdot x_{0}\right\}\right.$, and let $x_{0}^{\prime}$ be the point on the geodesic segment $\left[x_{0}, s_{0} \cdot x_{0}\right]$ at distance $\epsilon$ from $x_{0}$. Then, for every $s \in S_{0}$ we would have $\left(x_{0}^{\prime}, s^{-1} \cdot x_{0}\right)_{x_{0}}=d_{\mathcal{T}}\left(x_{0}, x_{0}^{\prime}\right)=\epsilon$ and $\left(x_{0}^{\prime}, s \cdot x_{0}^{\prime}\right)_{x_{0}}=d_{\mathcal{T}}\left(x_{0}, x_{0}^{\prime}\right)=\epsilon$ (because $\left.d\left(s \cdot x_{0}, s \cdot x_{0}^{\prime}\right)=\epsilon<d\left(s \cdot x_{0}, x_{0}\right)-\epsilon=L-\epsilon\right)$, hence

$$
\begin{aligned}
d_{\mathcal{T}}\left(x_{0}^{\prime}, s \cdot x_{0}^{\prime}\right) & =d_{\mathcal{T}}\left(x_{0}, s \cdot x_{0}\right)+2 d_{\mathcal{T}}\left(x_{0}, x_{0}^{\prime}\right)-2\left(x_{0}^{\prime}, s^{-1} \cdot x_{0}\right)_{x_{0}}-2\left(x_{0}^{\prime}, s \cdot x_{0}^{\prime}\right)_{x_{0}} \\
& <L-2 \epsilon .
\end{aligned}
$$

On the other hand, for every $s \in\left(S \cup S^{-1}\right) \backslash S_{0}$, we have, by the choice of $\epsilon$,

$$
d_{\mathcal{T}}\left(x_{0}^{\prime}, s \cdot x_{0}^{\prime}\right) \leq d_{\mathcal{T}}\left(x_{0}, s \cdot x_{0}\right)+2 \epsilon<L .
$$

Then, $x_{0}$ would not realise the minimal joint displacement of $S$, a contradiction. It follows that $\epsilon_{0}=0$ necessarily. We have therefore proved that there exists an element $s_{1} \in S \cup S^{-1}$ with $d_{\mathcal{T}}\left(x_{0}, s_{1} \cdot x_{0}\right)=L$, such that $x_{0}$ belongs to the interior of the geodesic segment $\left[s_{0} \cdot x_{0}, s_{1} \cdot x_{0}\right]$. In particular, $x_{0} \notin \mathrm{T}\left(s_{0}\right) \cup \mathrm{T}\left(s_{1}\right)$ (recall that by assumption, if $s_{0}$ or $s_{1}$ are hyperbolic, then they have translation length strictly smaller than $L$ ).

Then, the characteristic subset $\mathrm{T}\left(s_{1}\right)$ of $s_{1}$ intersects the segment $\left[x_{0}, s_{1} \cdot x_{0}\right]$ in a smaller subsegment containing the midpoint $m_{1}$ of $\left[x_{0}, s_{1} \cdot x_{0}\right]$; therefore, the point $s_{1}^{-1} \cdot x_{0}$ belongs to the connected component $\mathcal{T}_{1}$ of $\mathcal{T} \backslash\left\{x_{0}\right\}$ containing $m_{1}$. Analogously, $s_{0}^{-1} \cdot x_{0}$ belongs to the connected component $\mathcal{T}_{0}$ of $\mathcal{T} \backslash\left\{x_{0}\right\}$ containing the midpoint $m_{0}$ of $\left[x_{0}, s_{0} \cdot x_{0}\right]$. As $\mathcal{T}_{1} \neq \mathcal{T}_{0}$, it follows that

$$
d_{\mathcal{T}}\left(s_{0}^{-1} \cdot x_{0}, s_{1}^{-1} \cdot x_{0}\right)=d_{\mathcal{T}}\left(s_{0}^{-1} \cdot x_{0}, x_{0}\right)+d_{\mathcal{T}}\left(x_{0}, s_{1}^{-1} \cdot x_{0}\right)=d_{\mathcal{T}}\left(s_{0} \cdot x_{0}, s_{1} \cdot x_{0}\right) .
$$

We deduce that the four points $v_{0}=s_{0} \cdot x_{0}, v_{1}=x_{0}, w_{0}=s_{1} \cdot x_{0}$ and $w_{1}=s_{1} s_{0}^{-1} \cdot x_{0}$ are collinear; moreover, we have that $h=s_{1} s_{0}^{-1}$ sends $v_{0}$ in $w_{0}$ and $v_{1}$ in $w_{1}$, and that $d_{\mathcal{T}}\left(v_{0}, w_{0}\right)=d_{\mathcal{T}}\left(v_{1}, w_{1}\right)$, by (3). So, we apply Lemma 1.1(ii) and deduce that $h$ is hyperbolic, with axis passing through $v_{0}$ and $v_{1}=x_{0}$, and such that $\tau(h)=d_{\mathcal{T}}\left(v_{0}, h \cdot v_{0}\right)=d\left(s_{0} \cdot x_{0}, s_{1} \cdot x_{0}\right)=2 L$.

In what follows, we will set for short $\tau=\tau(h) \in[L, 2 L]$. Now, a suitable positive power of the hyperbolic element $h$ (for instance $h^{10+5 k}$ ) would satisfy all the required conditions, except for $(\mathrm{v})$. We will now show that there exists some (controlled) $n \geq 1$ and some $v \in S$ such that $g_{0}=h^{n} v$ is again a hyperbolic element satisfying (i)-(iv) and with oriented normalizer $N^{+}\left(g_{0}\right)=\left\langle g_{0}\right\rangle$.

Actually, as $G$ acts non-elementarily and without fixed points, there exists $v \in S$ which does not stabilize $\operatorname{Ax}(h)$. So, let $g=h^{n} v$. As $g \cdot \operatorname{Ax}(h)=\operatorname{Ax}\left(g h g^{-1}\right)$, we have $\operatorname{diam}(\operatorname{Ax}(h) \cap g \cdot \operatorname{Ax}(h)) \leq 2 \tau+k$ by $k$-acylindricity (Lemma 1.4). Since $h^{n} \cdot x_{0} \in \operatorname{Ax}(h)$ and $d_{\mathcal{T}}\left(g \cdot x_{0}, h^{n} \cdot x_{0}\right) \leq L$, we deduce that the projection on $\operatorname{Ax}(h)$ of any point of $g \cdot \operatorname{Ax}(h)$ falls at distance at most $2 \tau+L+k$ from $h^{n} \cdot x_{0}$. 
In particular, if $n \geq 6+k$, both $g h^{n} \cdot x_{0}$, which belongs to $g \cdot \operatorname{Ax}(h)$, and $g^{2} \cdot x_{0}$ project to the same point $y_{0} \in \operatorname{Ax}(h)$ with $d_{\mathcal{T}}\left(y_{0}, h^{n} \cdot x_{0}\right) \leq 2 \tau+L+k$ (because $d_{\mathcal{T}}\left(g h^{n} \cdot x_{0}, \operatorname{Ax}(h)\right) \geq n \tau-(5 L+k) \geq L$ and $\left.d_{\mathcal{T}}\left(g^{2} \cdot x_{0}, g h^{n} \cdot x_{0}\right) \leq L\right)$, see Figure 1. Then

$$
\begin{aligned}
d_{\mathcal{T}}\left(x_{0}, g^{2} \cdot x_{0}\right) & \geq d_{\mathcal{T}}\left(x_{0}, h^{n} \cdot x_{0}\right)+d_{\mathcal{T}}\left(h^{n} \cdot x_{0}, g^{2} \cdot x_{0}\right)-2 d_{\mathcal{T}}\left(y_{0}, h^{n} \cdot x_{0}\right) \\
& \geq n \tau+d_{\mathcal{T}}\left(x_{0}, v h^{n} v \cdot x_{0}\right)-2(2 \tau+L+k) \\
& \geq(2 n-4) \tau-4 L-2 k .
\end{aligned}
$$

On the other hand, $d_{\mathcal{T}}\left(x_{0}, g \cdot x_{0}\right) \leq d_{\mathcal{T}}\left(x_{0}, h^{n} \cdot x_{0}\right)+d_{\mathcal{T}}\left(x_{0}, v \cdot x_{0}\right) \leq n \tau+L$. Therefore, by Lemma 1.1(i), $g$ is hyperbolic for $n>9+2 k$ and

$$
\tau(g) \in[(n-9) \tau-2 k,(n+1) \tau] .
$$

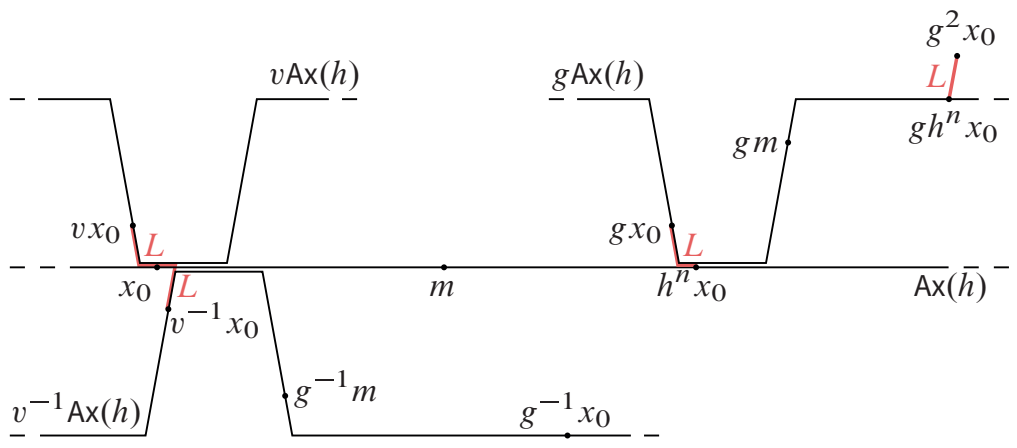

Figure 1. Configuration of $\operatorname{Ax}(h), v \cdot \operatorname{Ax}(h), v^{-1} \cdot \operatorname{Ax}(h)$ and $g \cdot \operatorname{Ax}(h)$

Now, notice that the midpoint $m$ of the segment $\left[x_{0}, g \cdot x_{0}\right]$ belongs to $\operatorname{Ax}(h)$ (as $\left.\tau\left(h^{n}\right) \geq L\right)$, hence the midpoint $g \cdot m$ of $\left[g \cdot x_{0}, g^{2} \cdot x_{0}\right]$ belongs to $g \cdot A x(h)$ and the midpoint $g^{-1} \cdot m$ of $\left[x_{0}, g^{-1} \cdot x_{0}\right]$ belongs to $v^{-1} \mathrm{Ax}(h)$. Moreover, we have seen that the projection to $\operatorname{Ax}(h)$ of any point of $g \cdot \operatorname{Ax}(h)$, in particular the projection $y_{0}^{\prime}$ of $g \cdot m$, falls at distance at most $2 \tau+L+k \leq 3 \tau+k$ from $h^{n} \cdot x_{0}$; similarly, the projection $x_{0}^{\prime}$ of $g^{-1} \cdot m$ to $\operatorname{Ax}(h)$ satisfies $d_{\mathcal{T}}\left(x_{0}, x_{0}^{\prime}\right) \leq 3 \tau+k$. Since by Lemma 1.1(i) the axis of $g$ contains $\left[g^{-1} \cdot m, m\right] \cup[m, g \cdot m]$, it follows that $\operatorname{Ax}(g)$ contains the segment $\left[x_{0}^{\prime}, y_{0}^{\prime}\right]$ of $\operatorname{Ax}(h)$, whose length is

$$
\ell\left(\left[x_{0}^{\prime}, y_{0}^{\prime}\right]\right) \geq d\left(x_{0}, h^{n} \cdot x_{0}\right)-d\left(x_{0}, x_{0}^{\prime}\right)-d\left(y_{0}, y_{0}^{\prime}\right) \geq(n-6) \tau-2 k
$$

and that

$$
d\left(x_{0}, \operatorname{Ax}(g)\right) \leq d\left(x_{0}, x_{0}^{\prime}\right) \leq 3 \tau+k .
$$

We can now show (iv). Recall that, by Lemma $1.3, N^{+}(g)$ is cyclic generated by some $\bar{g}$ of minimal translation length, so we may assume that $g=\bar{g}^{d}$ for some 
$d \geq 1$, with $\operatorname{Ax}(\bar{g})=\operatorname{Ax}(g)$. If $d>1$ then $\tau(\bar{g}) \leq \tau(g) / 2$, hence for $n>15+6 k$ we would obtain

$$
\begin{aligned}
\operatorname{diam}(\operatorname{Ax}(\bar{g}) \cap \operatorname{Ax}(h)) & \geq \ell\left(\left[x_{0}^{\prime}, y_{0}^{\prime}\right]\right) \\
& \geq(n-6) \tau-2 k \\
& >\frac{(n+1) \tau}{2}+\tau+k \\
& \geq \tau(\bar{g})+\tau+k,
\end{aligned}
$$

which, by Lemma 1.4, contradicts $k$-acilindricity. Therefore, setting $g_{0}=h^{n} v$ for $n=16+6 k$ we get $N^{+}\left(g_{0}\right)=\left\langle g_{0}\right\rangle$.

Notice that for this choice of $n$ we have $\left|g_{0}\right|_{S} \leq 2 n+1 \leq 33+12 k$; moreover, by (4) we have $\tau\left(g_{0}\right) \geq(7+6 k) \tau-2 k \geq 7+4 k$ and

$$
d_{\mathcal{T}}\left(x_{0}, s \cdot x_{0}\right) \leq L \leq \tau \leq \frac{1}{7+6 k}\left(\tau\left(g_{0}\right)+2 k\right)
$$

for all $s \in S$, so conditions (i)-(iii) hold. Finally, from (5) we deduce that $d_{\mathcal{T}}\left(x_{0}, \operatorname{Ax}\left(g_{0}\right)\right) \leq \frac{1}{7+6 k}\left(3 \tau\left(g_{0}\right)+13 k+6 k^{2}\right)$, so the lemma is proved.

Proof of Theorem 1. By Lemma 2.2, we can find $x_{0} \in \mathcal{T}$ and a hyperbolic element $g_{0} \in G$ with $N^{+}\left(g_{0}\right)=\left\langle g_{0}\right\rangle$ and satisfying the conditions (i)-(iv) of the lemma. Let now $v_{0}$ be a vertex with $d_{\mathcal{T}}\left(x_{0}, v_{0}\right) \leq \frac{1}{2}$. By Lemma $1.3, N\left(g_{0}\right)$ is isomorphic either to $N^{+}\left(g_{0}\right)$ or to the infinite dihedral group $N^{+}\left(g_{0}\right) \rtimes \mathbb{Z}_{2}$, generated by $g_{0}$ and by an elliptic element $\sigma_{0}$ of order two swapping $\operatorname{Ax}\left(g_{0}\right)$. Up to conjugating $\sigma_{0}$ by some power of $g_{0}$, we may assume that $\sigma_{0}$ fixes $v_{0}$. Now, for all $u \in S^{2}$ we have by (iii)

$$
d_{\mathcal{T}}\left(x_{0}, u \cdot x_{0}\right) \leq \frac{2}{7+6 k}\left(\tau\left(g_{0}\right)+2 k\right)<\tau\left(g_{0}\right),
$$

while, for all $g \in N\left(g_{0}\right)$ we have

$$
d_{\mathcal{T}}\left(x_{0}, g \cdot x_{0}\right) \geq d_{\mathcal{T}}\left(v_{0}, g \cdot v_{0}\right) \geq d_{\mathcal{T}}\left(\sigma_{0} \cdot v_{0}, g_{0} \cdot v_{0}\right) \geq \tau\left(g_{0}\right),
$$

unless $g \in\left\{1, \sigma_{0}\right\}$. So, $S^{2} \cap N\left(g_{0}\right) \subset\left\langle\sigma_{0}\right\rangle$. It follows that the map $s \mapsto \operatorname{Ax}\left(s g_{0} s^{-1}\right)$, for $s \in S$, is at most 2-to-1: in fact, if we have $\operatorname{Ax}\left(s g_{0} s^{-1}\right)=\operatorname{Ax}\left(s^{\prime} g_{0} s^{\prime-1}\right)$ then $s^{-1} s^{\prime} \in S^{2} \cap N\left(g_{0}\right)$, so $s=s^{\prime}$ or $s^{-1} s^{\prime}=\sigma_{0}$. Then, there exists a collection of conjugates $g_{i}=s_{i} g_{0} s_{i}^{-1}$, for $s_{i} \in S$ and $i \in I$, with mutually distinct axes and cardinality $|I| \geq|S| / 2$. Since $\operatorname{Ax}\left(g_{i}\right)=s_{i} \cdot \operatorname{Ax}\left(g_{0}\right)$ and $\tau\left(g_{i}\right)=\tau\left(g_{0}\right)$, by (iii) and (iv) we deduce that

$$
D:=d_{\mathcal{T}}\left(x_{0}, \operatorname{Ax}\left(g_{i}\right)\right) \leq d_{\mathcal{T}}\left(x_{0}, s_{i} \cdot x_{0}\right)+d_{\mathcal{T}}\left(x_{0}, \operatorname{Ax}\left(g_{0}\right)\right)<\frac{4 \tau\left(g_{0}\right)+15 k+6 k^{2}}{(7+6 k)} .
$$

Moreover, by $k$-acilindricity, we know that

$$
\Delta:=\operatorname{diam}\left(\operatorname{Ax}\left(g_{i}\right) \cap \operatorname{Ax}\left(g_{j}\right)\right) \leq 2 \tau\left(g_{0}\right)+k .
$$


It follows from Lemma 2.1 that for $p \geq 6$ the elements in $S_{0}=\left\{g_{i}^{p} \mid i \in I\right\}$ generate a free, Schottky subgroup $G_{0}<G$ of rank at least $|S| / 2$, since by (ii) we have

$$
\frac{2(D+\Delta)}{\tau\left(g_{0}\right)} \leq \frac{4\left(30 k^{2}+89 k+63\right)}{24 k^{2}+70 k+49}<6 .
$$

Also notice that choosing $p=6$ we have $S_{0} \subset S^{6(33+12 k)+2}=S^{200+72 k}$, by (i). Therefore, we deduce that

$$
\operatorname{Ent}(G, S) \geq \operatorname{Ent}\left(G_{0} \curvearrowright \mathcal{C}(G, S)\right) \geq \frac{1}{(200+72 k)} \operatorname{Ent}\left(G_{0}, S_{0}\right)
$$

and, as $G_{0}$ is free on $S_{0}$, which has cardinality at least $|S| / 2$, this yields the announced inequality.

\section{Applications}

Recall that, given a marked group $(G, S)$, a complete set of relators for $G$ is a finite subset $R$ of the free group on $S$ such that $G \cong \mathbb{F}(S) /\langle\langle R\rangle\rangle$, where $\langle\langle R\rangle\rangle$ denotes the normal subgroup generated by the elements in $R$. A first consequence of the entropy-cardinality inequality is a general, finiteness theorem for abstract groups admitting acylindrical splittings, which has an interest in its own.

Theorem 3.1. The number of isomorphism classes of marked groups $(G, S)$ admitting a k-acylindrical splitting and a complete set of relators of length at most $\ell$, satisfying $\operatorname{Ent}(G, S) \leq E$ is finite, bounded by an explicit function $N(k, \ell, E)$.

Proof of Theorem 3.1. The entropy-cardinality inequality yields a corresponding bound on the cardinality of $S$ in terms of the acylindricity constant and of $E$ :

$$
|S| \leq 1+e^{(200+72 k) E}=n(k, E) .
$$

Now, the number of possible presentations by relators of length smaller than $\ell$ on an alphabet $\mathcal{A}$ of at most $n$ letters is roughly bounded by $2^{n+n^{\ell}}$ (that is the number of subsets $S$ of $\mathcal{A}$, times the number of subsets $R$ of $\mathcal{A}^{\ell}$ ); this gives the announced bound of the number of marked groups $(G, S)$ by the function $N(k, \ell, E)=2^{n(k, E)+n(k, E)^{\ell}}$.

3.1. Quasiconvex groups of $\delta$-hyperbolic and CAT(0)-spaces. Recall that an action of a group $G$ on a $\delta$-hyperbolic or CAT( 0$)$-space $X$ is called quasi-convex if there exists an orbit $S=G x_{0}$ which is a $D$-quasi-convex subset of $X$ (i.e. all the geodesics joining two points $x_{1}, x_{2} \in S$ are included in the closed $D$-neighborhood of $S$ ), for some $D>0$. One also says that $G$ is a quasi-convex group of $X$. 
Proof of Corollary 2. Any marked $\delta$-hyperbolic group $(G, S)$ possesses a complete set of relators of length $\ell \leq(4 \delta+6)$ (see for instance [13], Chapter III. $\Gamma$, Proposition 2.2): the conclusion then immediately follows from Theorem 3.1.

Proof of Corollary 3. Let $G$ act on a proper, geodesic space $(X, d)$, and let $x_{0} \in$ $X$ a point with $D$-quasi-convex orbit. By a classical argument (Milnor-Švarc Lemma), the set $S=\left\{g \in G \mid d\left(x_{0}, g \cdot x_{0}\right) \leq 2 D+1\right\}$ generates $G$. Actually, for every $g \in G$, consider a geodesic $c:[0, \ell] \rightarrow X$ from $x_{0}$ to $g . x_{0}$, and orbit points $g(k) . x_{0}$ such that $g(0)=1, g(\lceil\ell\rceil)=g$ and $d\left(c(k), g(k) \cdot x_{0}\right) \leq D$, given by the condition of $D$-quasiconvexity. Then, setting $\gamma(1)=g(1)$ and $\gamma(k)=$ $g(k-1)^{-1} g(k)$, one has that the $\gamma(k)$ 's are in $S$ and $g=\gamma(1) \cdot \gamma(2) \cdots \gamma(\lceil\ell\rceil)$. By construction, we also have

$$
\frac{1}{2 D+1} d\left(x_{0}, g \cdot x_{0}\right) \leq|g|_{S} \leq d\left(x_{0}, g \cdot x_{0}\right)+1 ;
$$

therefore the marked group $(G, S)$ is $(2 D+1,1)$-quasi-isometric to the orbit $G . x_{0}$, endowed with the distance $d$ induced by $X$; it follows that, in case (i), $(G, S)$ is $\delta^{\prime}$-hyperbolic, for some $\delta^{\prime}=\delta^{\prime}(\delta, D)$. On the other hand, from the left-hand side of (6) we deduce that

$$
\operatorname{Ent}(G, S) \leq(2 D+1) \cdot \operatorname{Ent}(G \curvearrowright X) \leq(2 D+1) E .
$$

The conclusion in case (i) then follows from Corollary 2.

In case (ii), we proceed similarly to [13], Chapter III. $\Gamma$, Proposition 2.2, by replacing the cocompactness assumption by quasiconvexity. Namely, given $S$ as above, one decomposes any relation $r=s_{1} \cdots s_{n}$ on $S$ for $G$ as a product of relations

$$
r=\prod_{i=1}^{n-1}\left(\sigma_{i} s_{i+1} \sigma_{i+1}^{-1}\right),
$$

where $\sigma_{i}:=s_{1} \cdots s_{i}$, and then shows that each relation $\sigma_{i} s_{i+1} \sigma_{i+1}^{-1}$ is product of conjugates of words $r_{k}$ of $S$-length at most $8 D+6$. Actually, choose again geodesics $c_{i}:\left[0, \ell_{i}\right] \rightarrow X$ from $x_{0}$ to $\sigma_{i} . x_{0}$, and then orbit points $g_{i}(k) . x_{0}$ with $g_{i}(0)=1, g_{i}\left(\left\lceil\ell_{i}\right\rceil\right)=\sigma_{i}$ for all $i$ and $d\left(c_{i}(k), g_{i}(k) . x_{0}\right) \leq D$, provided by the $D$-quasiconvexity. We then consider the elements $\gamma_{i}(k):=g_{i}(k)^{-1} g_{i}(k+1)$ and $\mu_{i}(k):=g_{i}(k)^{-1} g_{i+1}(k)$ of $G$, and notice that

$$
d\left(x_{0}, \gamma_{i}(k) \cdot x_{0}\right) \leq 2 D+d\left(c_{i}(k), c_{i}(k+1)\right)=2 D+1 .
$$

and that, by the convexity of the metric of $X$,

$d\left(x_{0}, \mu_{i}(k) . x_{0}\right) \leq 2 D+d\left(c_{i}(k), c_{i+1}(k)\right) \leq 2 D+d\left(\sigma_{i} \cdot x_{0}, \sigma_{i+1} \cdot x_{0}\right)=4 D+1$.

Therefore, $\gamma_{i}(k) \in S$ and $\left|\mu_{i}(k)\right|_{S} \leq 4 D+2$, by (6); so, $\mu_{i}(k)$ can be represented by a word $\tilde{\mu}_{i}(k)$ on $S$ of length $\leq 4 D+2$. Accordingly, all the relations $\sigma_{i} s_{i+1} \sigma_{i+1}^{-1}$ 
can be decomposed as products of conjugates

$$
\begin{aligned}
\sigma_{i} s_{i+1} \sigma_{i+1}^{-1}= & g_{i}\left(\left\lceil\ell_{i}\right\rceil\right) r_{i}\left(\left\lceil\ell_{i}\right\rceil\right) g_{i}\left(\left\lceil\ell_{i}\right\rceil\right)^{-1} \ldots \\
& g_{i}(k) r_{i}(k) g_{i}(k)^{-1} \ldots \\
& g_{i}(1) r_{i}(1) g_{i}(1)^{-1},
\end{aligned}
$$

where the words $r_{i}(k):=\tilde{\mu}_{i}(k+1) \gamma_{i+1}(k)^{-1} \tilde{\mu}_{i}(k)^{-1} \gamma_{i}(k)$ represent relators on $S$ whose $S$-lengths do not exceed $8 D+6$. The conclusion for case (ii) then follows from Theorem 3.1.

3.2. CAT(0)-spaces with negatively curved splittings. We say that a locally CAT(0)-space $X$ admits a splitting if $X$ is isometric to the gluing $X_{1} \sqcup_{\phi} X_{2}$ of two locally CAT(0)-spaces $X_{1}, X_{2}$ along compact, locally convex, isometric subspaces $Z_{i} \hookrightarrow X_{i}$ via an isometry $\phi: Z_{1} \rightarrow Z_{2}$; or if $X$ is isometric to the space $X_{0} \sqcup_{\phi}$ obtained by identifying two such subspaces $Z_{i} \subset X_{0}$ to each other by an isometry $\phi$. We say that the splitting is non-trivial if $\pi_{1}(X)$ corrispondingly splits as a HNN-extension or a non-trivial amalgamated product.

Notice that the space obtained by such gluings is always locally CAT(0) (cf. [13], Proposition 11.6). We will say that the splitting is negatively curved if the subspace $Z$ of $X$ obtained by identifying $Z_{1}$ to $Z_{2}$ has a neighbourhood $U(Z)$ in $X$ such that $U(Z) \backslash Z$ is a locally CAT $(-k)$-space for some $k>0$.

The following fact is crucial to prove acylindricity for negatively curved splittings of CAT(0)-spaces, and we believe it is folklore; we will give a proof of it in Appendix C, for completeness.

Proposition C.1. Let $Z$ be a compact, locally convex subspace of a compact, complete locally $\mathrm{CAT}(0)$-space $X$. Assume that $X$ is negatively curved around $Z$. then, $H=\pi_{1}(Z)$ is malnormal in $G=\pi_{1}(X)$.

Proof of Theorem 4. If $X$ admits a non-trivial, negatively curved splitting $X=$ $X_{1} \sqcup_{Z} X_{2}$ or $X=X_{0} \sqcup_{\phi}$, along two isometric, locally convex subspaces $Z_{i} \cong Z$ identified via an isometry $\phi$, then $\pi_{1}(X)$ splits as a non-trivial amalgamated product of the groups $G_{i}=\pi_{1}\left(X_{i}\right)$ over $H=\pi_{1}(Z)$, or as a HNN-extension of $G_{0}=\pi_{1}\left(X_{0}\right)$ along subgroups $H_{i}=\pi_{1}\left(Z_{i}\right)$, via an isomorphism $\phi_{*}: H_{1} \rightarrow H_{2}$.

Now, the subgroup $H$ is malnormal in $G=\pi_{1}(X)$, by Proposition C.1. Therefore, in the amalgamated case, if $\iota_{G_{i}}: H \hookrightarrow G_{i}$ are the natural inclusions, the subgroups $H_{i}=\iota_{G_{i}}(H)$ are malnormal in each $G_{i}$. So, either $H=1$, and $G$ admits a 0 -acylindrical splitting (being a non-trivial free product), or $\left[G_{i}: H_{i}\right]>2$ (otherwise $H_{i}$ would be normal and malnormal in $G_{i}$, hence trivial); in this last case, $G$ admits a 1-acylindrical splitting, by Lemma 1.6(ii). When $G=G_{0} *_{\varphi}$, since $H$ is malnormal in $G$ then the subgroups $H_{i}$ are conjugately separated and malnormal in $G_{0}$; in particular, there exists a $H_{i} \subsetneq G_{0}$, hence $G$ admits a 1-acylindrical splitting, by Lemma 1.7. 
By Corollary 3 , we deduce that $\pi_{1}(X)$ belongs to a finite class of groups, whose number is bounded by a universal function of $E$ and $D$. Since the locally CAT(0)-spaces are aspherical, we can conclude by Whitehead's theorem the finiteness of the homotopy types.

Proof of Corollary 5. It follows from the fact that, in dimension greater than 4 , the homeomorphism type of closed, non-positively curved manifolds is determined by their homotopy type, from the solution of the Borel Conjecture by Bartels and Lück for CAT(0)-manifolds [7]. Moreover, the works of Kirby and Siebenmann [37] and of Hirsch and Mazur [33] on PL structures and their smoothings imply the finiteness of smooth structures in dimension $n \geq 5$ (cf. also [39], Theorem 7.2).

The fact that the fundamental group determines the diffeomorphism type is well known in dimension 2, and follows in dimension 3 from Perelman's solution of the geometrization conjecture (we now know that any closed, negatively curved 3-manifold also admits a hyperbolic metric; so Mostow's rigidity applies).

\section{Examples}

4.1. Two-dimensional orbifolds. We recall shortly some basic facts about orbifolds; for a primer concerning 2-dimensional orbifolds we refer to [58] and [65]. Following Thurston [65], a $n$-dimensional orbifold $\mathcal{O}$ (without boundary) is a Hausdorff, paracompact space which is locally homeomorphic either to $\mathbb{R}^{n}$, or to the quotient of $\mathbb{R}^{n}$ by a finite group action; similarly, $n$-orbifolds with boundary also have points whose neighbourhood is homeomorphic to the quotient of the half-space $\mathbb{R}_{+}^{n}$ by a finite group action. For the sake of simplicity we shall consider uniquely compact 2-dimensional orbifolds with conical singular points, that is points which have a neighborhood modelled on the quotient of $D^{2}$ by a finite cyclic group. Nevertheless, it follows from the description of singularities in [58] and [65] that given a general compact 2-orbifold there exists a canonically constructed double cover which has only conical singularities; this cover is obtained by doubling the underlying space along the reflector lines, duplicating the conical singular points and transforming the so called corner reflectors into conical singular points. An orbifold is good if it can be covered by a genuine surface, and bad otherwise; the (finite) list of bad orbifolds can be found, for instance, in [65] (Theorem 13.3.6).

We shall denote by $\mathcal{O}=\mathcal{O}\left(g, h ; p_{1}, \ldots, p_{k}\right)$ the compact 2-orbifold having as underlying topological space a compact surface $|\mathcal{O}|$ of genus $g \in \mathbb{Z}$ (using negative values for the genus of non-orientable surfaces), $h$ boundary components and $k$ singular points $x_{1}, \ldots, x_{k}$ of orders $p_{1}, \ldots, p_{k}$. By the classification of compact 2-orbifolds given by Thurston in [65], Chapter 13, an orbifold with conical singular points is completely determined by its underlying topological 
space together with the number and the orders of its singular points. In view of this fact, we shall say that two smooth compact 2-orbifolds with conical singularities are isomorphic if they have the same underlying surface, the same number of singular points, and the same order at each singular point, up to permutations.

For a formal definition of the orbifold fundamental group, we refer to [9]; to our purposes, it will be sufficient to recall that the orbifold fundamental group of $\mathcal{O}=\mathcal{O}\left(g, h ; p_{1}, \ldots, p_{k}\right)$ admits one of the following presentations:

$$
\begin{aligned}
\pi_{1}^{\mathrm{orb}}(\mathcal{O})= & \left\langle a_{1}, b_{1}, \ldots, a_{g}, b_{g}, c_{1}, \ldots, c_{k}, d_{1}, \ldots, d_{h}\right| \\
& \left.\prod_{i=1}^{g}\left[a_{i}, b_{i}\right] \cdot \prod_{j=1}^{k} c_{j} \cdot \prod_{\ell=1}^{h} d_{\ell}=1, c_{i}^{p_{i}}=1\right\rangle, \\
\pi_{1}^{\mathrm{orb}}(\mathcal{O})= & \left\langle a_{1}, \ldots, a_{|g|}, c_{1}, \ldots, c_{k}, d_{1}, \ldots, d_{h}\right| \\
& \left.\prod_{i=1}^{|g|} a_{i}^{2} \cdot \prod_{j=1}^{k} c_{j} \cdot \prod_{\ell=1}^{h} d_{\ell}=1, c_{i}^{p_{i}}=1\right\rangle,
\end{aligned}
$$

depending on whether the genus is positive or negative. The generators $a_{i}$ 's, $b_{j}$ 's, $d_{\ell}$ 's are the fundamental system of generators of $|\mathcal{O}|$, with $d_{\ell}$ corresponding to the $l$-th boundary component, whereas the $c_{i}$ 's represent the generators of the isotropy groups associated to the singular points of $\mathcal{O}$.

The usual Euler characteristic can be generalized in a natural way to the case of compact 2-orbifolds [58], and in the case we are considering the formula reads

$$
\begin{aligned}
\chi_{\operatorname{orb}}\left(\mathcal{O}\left(g, h ; p_{1}, \ldots, p_{k}\right)\right) & =\chi(|\mathcal{O}|)-\sum_{i=1}^{k}\left(1-\frac{1}{p_{i}}\right) \\
& =\left\{\begin{array}{l}
2-2 g-h-\sum_{i=1}^{k}\left(1-\frac{1}{p_{i}}\right), \\
2-|g|-h-\sum_{i=1}^{k}\left(1-\frac{1}{p_{i}}\right),
\end{array}\right.
\end{aligned}
$$

depending on the sign of the genus $g$. The orbifold Euler characteristic determines, for a good orbifold $\mathcal{O}$, the kind of geometric structure that can be given to $\mathcal{O}$ : that is, whether its universal cover $\widetilde{\mathcal{O}}-$ which is a simply connected surface - admits a $\pi_{1}^{\text {orb }}(\mathcal{O})$-invariant spherical, flat or hyperbolic Riemannian metric. We shall accordingly call compact 2-orbifolds with negative Euler characteristic orbifolds of hyperbolic type.

Among compact 2-orbifolds of hyperbolic type there is a particular family whose groups are generated by symmetries of hyperbolic triangles along their 
edges: these groups are called triangle groups, and the corresponding orbifolds are those of the form $\mathcal{O}(0,0 ; p, q, r)$, with $p=2, q=3, r \geq 5$ or with $p, q, r \geq 3$ and at least one of them strictly greater than 3 .

In Theorem 6 we consider general Riemannian 2-orbifolds: that is, good compact 2-orbifolds $\mathcal{O}$ (with conical singularities) whose orbifold universal cover $\widetilde{\mathcal{O}}$ is endowed with any $\pi_{1}^{\text {orb }}(\mathcal{O})$-invariant Riemannian metric (that is, not necessarily with constant curvature). The entropy of $\mathcal{O}$ is correspondingly defined, as explained in the introduction, as $\operatorname{Ent}(\mathcal{O})=\operatorname{Ent}\left(\pi_{1}^{\mathrm{orb}}(\mathcal{O}) \curvearrowright \widetilde{\mathcal{O}}\right)$.

Notice that compact, two-dimensional orbifolds with non-negative orbifold Euler characteristic yield only finitely many isomorphism classes, without any assumption on their entropy and diameter, as it can be easily checked from the Euler characteristic formula.

The proof Theorem 6 will then split in two separate cases:

(a) non-triangular, Riemannian 2-orbifolds of hyperbolic type;

(b) triangular 2-orbifolds of hyperbolic type.

In fact, in the first case, the orbifold groups admit a 2-acylindrical splitting, as we will show in detail in Proposition A.1 of Appendix A, and we can use the Entropycardinality inequality. In the second case, acylindrical splittings are not available for such groups, and we will need an ad-hoc computation to conclude. We will denote by $\mathcal{O}_{h, n t}^{2}(E, D)$ and $\mathcal{O}_{h, t}^{2}(E, D)$ the classes of compact, Riemannian 2-orbifolds of negative orbifold Euler characteristic with entropy and diameter bounded by $E$ and $D$, which fall, respectively, in cases (a) and (b).

Proof of finiteness of isomorphism types in $\mathcal{O}_{h, n t}^{2}(E, D)$. The orbifold groups in this class admit a 2-acylindrical splitting, cf. Proposition A.1 of Appendix A. Then, for any orbifold $\mathcal{O} \in \mathcal{O}_{h, n t}^{2}(E, D)$ we choose $\tilde{x}_{0} \in \widetilde{\mathcal{O}}$, and apply the following, classical result (cf. [60]) to $G=\pi_{1}^{\text {orb }}(\mathcal{O})$ and to the open ball $U=$ $B_{\widetilde{\mathcal{O}}}\left(\tilde{x}_{0}, M\right)$ of $\widetilde{\mathcal{O}}$, for $M=D+\epsilon$ and arbitrary $\epsilon>0$.

Lemma 4.1. Let $G$ act by homeomorphisms on a path-connected, simply connected topological space $X$, and let $U$ be a path-connected open set such that $G . U=X$. Let

$$
S=\{s \in G \mid s U \cap U \neq \varnothing\}
$$

and

$$
T=\left\{\left(s_{1}, s_{2}\right) \in S \times S \mid U \cap\left(s_{1} U\right) \cap\left(s_{1} s_{2} U\right) \neq \emptyset\right\} .
$$

Then $S$ generates $G$ and one has $G \cong F(\Sigma) /\langle\langle\Theta\rangle\rangle$ where $\Sigma$ is the set of symbols $\left\{x_{s} \mid s \in S\right\}$, and $\Theta$ is the set of words on $S \cup S^{-1}$ given by

$$
\left\{x_{s_{1}} x_{s_{2}} x_{s_{1} s_{2}}^{-1} \mid\left(s_{1}, s_{2}\right) \in T\right\} .
$$

(Notice that if $\left(s_{1}, s_{2}\right) \in T$, then $s_{1} s_{2} \in S$ so $x_{s_{1} s_{2}}^{-1}$ makes sense). 
The lemma yields a finite generating set $S_{M}$ of $G$, such that $d\left(x_{0}, g \cdot x_{0}\right)<M$ for all $g \in S_{M}$, which we call $M$-short generators of $G$ at $x_{0}$, and a triangular presentation of the group: that is, such that the group of relations is generated, as a normal subgroup of $\mathbb{F}\left(S_{M}\right)$, by relators of length at most 3 . Since we have $d\left(x_{0}, g \cdot x_{0}\right) \leq M \cdot|g|_{S_{M}}$, we get $\operatorname{Ent}\left(\pi_{1}(\mathcal{O}), S_{M}\right) \leq M \operatorname{Ent}(\mathcal{O}) \leq M E$. Letting $\epsilon \rightarrow 0$, we deduce from Theorem 1 that $\left|S_{M}\right| \leq 1+e^{344 D E}=N(E, D)$.

As in the proof of Theorem 3.1 observe now that the number of possible triangular presentations that can be build with letters from some subset $S$ of an alphabet of cardinality $N$ does not exceed $2^{N+N^{3}}$; therefore, the number of such orbifold groups is bounded from above by $2^{N(E, D)}+2^{N(E, D)^{3}}$. To conclude, remark that $\pi_{1}^{\text {orb }}(\mathcal{O})$ determines the isomorphism class of $\mathcal{O}$ for closed orbifolds, while for orbifolds with boundary the isomorphism class is determined by the orbifold group and the number of boundary components; in any case, there are a finite number of non-isomorphic 2-orbifolds for any given group $\pi_{1}^{\text {orb }}(\mathcal{O})$.

Proof of finiteness of isomorphism types in $\mathcal{O}_{h, t}^{2}(E, D)$. Call

$$
G_{p, q, r}=\pi_{1}^{\mathrm{orb}}(\mathcal{O}(0,0 ; p, q, r))
$$

the triangle orbifold group, with $3 \leq p \leq q \leq r$, and $r \geq 4$. By the canonical presentations of the compact 2-orbifold groups recalled before, we know that $G_{p, q, r}$ is isomorphic to $\left.\mathbb{Z}_{p} * \mathbb{Z}_{r} /\left\langle(a c)^{q}\right\rangle\right\rangle$, where $a, c$ are, respectively, generators of $\mathbb{Z}_{p}$ and $\mathbb{Z}_{r}$. Moreover, since the diameter of the Riemannian orbifold $\mathcal{O}(0,0 ; p, q, r))$ is bounded by $D$, we may assume that the fixed points $A, C$ respectively of $a$ and $c$ satisfy $d(A, C) \leq D$. We will now show that the value of $r$ is bounded in terms of $D$ and $E$, by evaluating the Poincaré series of $G_{p, q, r}$ at $C$ :

Lemma 4.2. Let

$$
G_{p, q, r}=\left\langle a, b, c \mid a b c=a^{p}=b^{q}=c^{r}=1\right\rangle,
$$

where $3 \leq p \leq q \leq r$ and $r \geq 4$, be the fundamental group of a triangular orbifold of hyperbolic type. The following set of elements of $\mathbb{Z}_{p} * \mathbb{Z}_{r}$ naturally injects into $G_{p, q, r}$ :

$$
\mathcal{W}_{r}=\left\{c^{j_{1}} a c^{j_{2}} a \cdots a c^{j_{m}} \mid m \in \mathbb{N}, j_{k} \text { even, } 2 \leq j_{k} \leq r-2\right\}
$$

(by a slight abuse of notation, we will use $\mathcal{W}_{r}$ and the reduced forms $c^{j_{1}} a \cdots a c^{j_{m}}$ to denote both the elements of $\mathbb{Z}_{p} * \mathbb{Z}_{r}$ and their images in $\left.G_{p, q, r}\right)$.

Assuming this lemma for a moment, we can compute

$$
P_{s}\left(G_{p, q, r}, C\right) \geq \sum_{g \in \mathcal{W}_{r}} e^{-s d(C, g \cdot C)}=\sum_{m>0} \sum_{1 \leq i_{1}, \ldots, i_{m} \leq \frac{r-2}{2}} e^{-s d\left(C, c^{2 i_{1}} a \cdots a c^{2 i_{m}} \cdot C\right)} .
$$


Now, $d\left(C, c^{2 i_{1}} a \cdots c^{2 i_{m}} \cdot C\right) \leq(m-1) d(C, a \cdot C)+\sum_{k=1}^{m} d\left(C, c^{2 i_{k}} \cdot C\right) \leq 2 m D$, which plugged into (7) yields

$$
P_{S}\left(G_{p, q, r}, C\right) \geq \sum_{m>0} \sum_{1 \leq i_{1}, \ldots, i_{m} \leq \frac{r-2}{2}} e^{-2 s m D} \geq \sum_{m>0}\left(\left\lfloor\frac{r}{2}-1\right\rfloor e^{-2 s D}\right)^{m} .
$$

Since $P_{s}\left(G_{p, q, r}, C\right)$ converges for all $s>E$, this shows that $\left\lfloor\frac{r}{2}-1\right\rfloor \leq e^{2 E D}$.

As $r \geq q \geq p$ this proves the finiteness of the fundamental groups and of isomorphisms classes of triangular orbifolds with bounded entropy and diameter.

Proof of Lemma 4.2. We know that $G_{p, q, r}$ is isomorphic to $\left.\mathbb{Z}_{p} * \mathbb{Z}_{r} /\left\langle(a c)^{q}\right\rangle\right\rangle$, where $a, c$ are, respectively, generators of $\mathbb{Z}_{p}$ and $\mathbb{Z}_{r}$. We start with the case $q \geq 4$.

Recall that a word on $S=\left\{a, a^{-1}, c, c^{-1}\right\}$, representing an element of $\mathbb{Z}_{p} * \mathbb{Z}_{r}$ with normal form $w=a^{p_{1}} c^{q_{1}} \ldots a^{p_{n}} c^{q_{n}}$, possibly with $p_{1}=0$ or $q_{n}=0$, is called weakly cyclically reduced if the last syllable of $w$ (that is, $c^{q_{n}}$, if $q_{n} \neq 0$ ) is different from the first one. If $R$ denotes the set of weakly cyclically reduced conjugates of $(c a)^{ \pm q}$, then the presentation of $G_{p, q, r}$ as $\mathbb{Z}_{p} * \mathbb{Z}_{r} /\langle\langle R\rangle\rangle$ satisfies the $C^{\prime}\left(\frac{1}{6}\right)$ condition for free products, see [41], Chapter V, $\$ 9$ (i.e., every $r \in R$ has syllable length $>6$ and every prefix of an element $r \in R$ which is a piece $e^{6}$ has syllable length $<\frac{1}{6} \ell(r)$ ).

It follows from small cancellation theory (Greendlinger Lemma, Chapter V, Theorem 9.3 in [41]) that any element $w \in \mathbb{Z}_{p} * \mathbb{Z}_{q}$ belonging to $\langle\langle R\rangle\rangle$ has a normal form which contains as a subword a prefix $r_{0}$ of some $r \in R$ of syllable length $\ell\left(r_{0}\right) \geq 5$.

It is then straightforward to check that, by construction, if $w, w^{\prime} \in \mathcal{W}_{r}$ then the normal form of $w w^{\prime-1}$ does not contain any such $r_{0}$ as a subword, unless $w=w^{\prime}$ (actually, after possible cancellation and consolidation, the normal form of $w w^{\prime-1}$ becomes $c^{j_{1}} a \cdots a c^{2 k} a^{-1} \cdots a^{-1} c^{-j_{1}^{\prime}}$ for some $k \neq 0$, and it does not contain any of the subwords acac, caca, $a^{-1} c^{-1} a^{-1} c^{-1}, c^{-1} a^{-1} c^{-1} a^{-1}$, since $a \neq a^{-1}$ and $c^{j_{k}} \neq c$ ). It follows that $\mathcal{W}_{r}$ injects into $G_{p, q, r}$.

When $p=q=3$, the above presentation of $G_{3,3, r}$ does not satisfy neither condition $C^{\prime}\left(\frac{1}{6}\right)$ for free products, nor the other classical small cancellation conditions under which the Greendlinger Lemma is available. However, these groups have nice geometric representations in $\operatorname{Isom}\left(\mathbb{H}^{2}\right)$ and their Cayley graphs can be seen as the 1-skeleton of uniform tilings of $\mathbb{H}^{2}$ : we recall briefly their construction, which we will use to show that the subset $\mathcal{W}_{r}$ injects, and refer to [47] for details.

\footnotetext{
${ }^{6}$ Notice that the small cancellation theory on free products differs from general cancellation theory, the relevant length and notion of piece being given by the syllable length and by the subdivision in syllables provided by the normal form.
} 
Consider a hyperbolic triangle $\mathcal{T}=A B C$ with interior angles $\alpha=\beta=\frac{\pi}{3}$ and $\gamma=\frac{\pi}{r}$ respectively at the vertices $A, B$ and $C$ : the group $G_{3,3, r}$ is generated by the rotations $a, b, c$ by $2 \alpha, 2 \beta, 2 \gamma$ centered at the vertices $A, B, C$, with $b=c a$. It is well known that $G_{3,3, r}$ is a subgroup of index two of the Coxeter group $\widehat{G}_{3,3, r}$, generated by the reflexions $a^{\prime}, b^{\prime}, c^{\prime}$ with respect to the edges $B C, C A$ and $A B$. Let $\mathcal{G}_{a c}$ be the Cayley graph of $G_{3,3, r}$ with respect to the generating set $\{a, c\}$. To embed $\mathcal{G}_{a c}$ in $\mathbb{H}^{2}$, start by constructing the (irregular) hexagon $\mathcal{H}_{b}$ obtained by applying all the rotations $b^{k}$ to $Q_{a}=\mathcal{T} \cup a^{\prime} \mathcal{T}$ : the hexagon $\mathcal{H}_{b}$, under the action of $G_{3,3, r}$, yields a regular tiling $\mathcal{P}_{b}$ of $\mathbb{H}^{2}$, see Figure 2(i). The derived tiling $\mathcal{P}_{b}^{\prime}$ has for tiles the polygons whose vertices are the middle points of the edges of $\mathcal{P}_{b}$ issuing from a common vertex: it is a hyperbolic tiling by triangles, hexagons and $r$-gones whose boundary $\partial \mathcal{P}_{b}^{\prime}$ is a graph isomorphic to $\mathcal{G}_{a c}$, as proved in [47], see Figure 2(ii). Namely, choosing a vertex of $\mathcal{P}_{b}^{\prime}$ as origin, say the midpoint $O$ of the edge $A C$, then all the edges of $\mathcal{P}_{b}^{\prime}$ can be coherently oriented and labelled by $\{a, c\}$ so that there exists a natural isomorphism between $\partial \mathcal{P}_{b}^{\prime}$ and $\mathcal{G}_{a c}$, preserving the edge labels and orientations, and sending $O$ to the basepoint $e$ of the Cayley graph $\mathcal{G}_{a c}$.

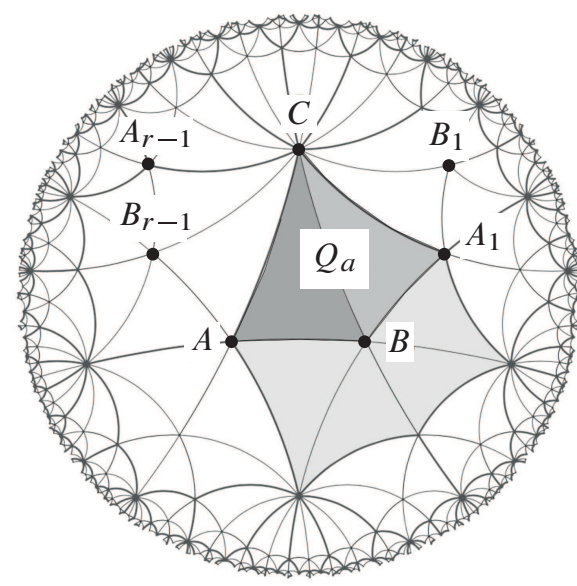

(i) the tiling $\mathcal{P}_{b}$

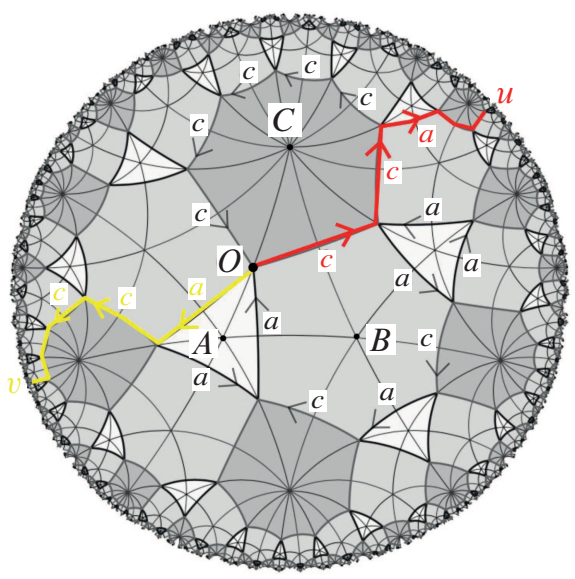

(ii) the tiling $\mathcal{P}_{b}^{\prime}$ with $\partial \mathcal{P}_{b}^{\prime} \cong \mathcal{G}_{a c}$

Figure 2

Consider now the $2 r$-gone $\mathcal{H}_{c}$ obtained by applying all the rotations $c^{k}$ to $Q_{a}$, with vertices $A_{k}=c^{k} \cdot A$ and $B_{k}=c^{k} \cdot B$. Notice that the geodesics supporting two edges of $\mathcal{H}_{c}$ intersect if and only if they are adjacent or separated by one edge. Indeed, denoting by $\sigma_{P Q}$ the bi-infinite geodesic containing $P Q$, then clearly $\sigma_{A_{i} B_{i}}$ and $\sigma_{A B}$ concur in the vertex of a triangle for $i=1, r-1$. On the other hand, if $\sigma_{A_{i} B_{i-1}}$ or $\sigma_{A_{i} B_{i}}$ intersected $\sigma_{A B}$ beyond $B$ for some $1<i<r$, then also the geodesic $\sigma_{C A_{1}}$ would intersect $\sigma_{A B}$, which is impossible since the opposite edges 
$C A_{1}$ and $A B$ of $Q_{a}$ lie on ultraparallel geodesics (they form with $A_{1} B$ interior angles whose sum is $\pi$ ); a similar argument shows that neither $\sigma_{A_{i} B_{i-1}}$ nor $\sigma_{A_{i} B_{i}}$ can intersect $\sigma_{A B}$ beyond $A$, for $i<r-1$.

Let now $\mathcal{C}\left(\sigma_{A C}^{+}, \frac{\pi}{3}\right)$ be the open geodesic cone whose axis is the geodesic ray $\sigma_{A C}^{+}$issuing from $A$ and containing $C$, with opening angle $\frac{\pi}{3}$,

$$
w \cdot O \in \mathcal{C}\left(\sigma_{A C}^{+}, \frac{\pi}{3}\right) \quad \text { for every } w=c^{j_{1}} a \cdots a c^{j_{m}} \in \mathcal{W}_{r} .
$$

We will prove this assertion by induction on $m$. The assertion is evident for $m=1$, so assume that it holds true for $m-1$, and let $w=c^{j_{1}} a \cdots a c^{j_{m}} \in \mathcal{W}_{r}$.

Now, the cone $\mathcal{C}\left(\sigma_{A C}^{+}, \frac{\pi}{3}\right)$ is bounded by the rays $\sigma_{A B}^{+}$and $\sigma_{A B_{r-1}}^{+}$; on the other hand, since $a \cdot \mathcal{C}\left(\sigma_{A C}^{+}, \frac{\pi}{3}\right) \subset \mathbb{H}^{2} \backslash \overline{\mathcal{C}\left(\sigma_{A C}^{+}, \frac{\pi}{3}\right)}$, the cone $c^{j_{1}} a \cdot \mathcal{C}\left(\sigma_{A C}^{+}, \frac{\pi}{3}\right)$ is included in the cone with opening angle $\frac{2 \pi}{3}$ bounded by the geodesic rays $\sigma_{A_{j_{1}} B_{j_{1}-1}}^{+}, \sigma_{A_{j_{1}} B_{j_{1}}}^{+}$issued from $A_{j_{1}}$, supporting the edges $A_{j_{1}} B_{j_{1}-1}, A_{j_{1}} B_{j_{1}}$ of $\mathcal{H}_{c}$. We already saw that the geodesics supporting these edges do not intersect $\sigma_{A B}$ unless $j_{1}=1$ or $j_{1}=r-1$, therefore the cone $c^{j_{1}} a \cdot \mathcal{C}\left(\sigma_{A C}^{+}, \frac{\pi}{3}\right)$ is included in $\mathrm{e}\left(\sigma_{A C}^{+}, \frac{\pi}{3}\right)$, for our choice of $j_{1}$. Moreover, by the induction hypothesis, we have that $c^{j_{2}} a \cdots a c^{j_{m}} \cdot O \in \mathcal{C}\left(\sigma_{A C}^{+}, \frac{\pi}{3}\right)$. It then follows that

$$
w \cdot O \in c^{j_{1}} a \cdot \mathcal{C}\left(\sigma_{A C}^{+}, \frac{\pi}{3}\right) \subset \mathcal{C}\left(\sigma_{A C}^{+}, \frac{\pi}{3}\right),
$$

which proves $(\star)$.

Let us finally show that $\mathcal{W}_{r}$ injects in $G_{3,3, r}$. Given two words $w=c^{j_{1}} a \cdots a c^{j_{m}}$ and $w^{\prime}=c^{j_{1}^{\prime}} a \cdots a c^{j_{m^{\prime}}^{\prime}}$ in $\mathcal{W}_{r}$, by induction on $\max \left\{m, m^{\prime}\right\}$ it is enough to show that if $w=w^{\prime}$ in $G_{3,3, r}$ then $j_{1}=j_{1}^{\prime}$. So, assume that $j_{1}>j_{1}^{\prime}$, and let $u=c^{j} a c^{j_{2}} a \cdots a c^{j_{m}}$ and $v=a c^{j_{2}^{\prime}} a \cdots a c^{j_{m^{\prime}}^{\prime}}$, with $j=j_{1}-j_{1}^{\prime}$ and $2 \leq j \leq r-2$ (as $j_{1}$ and $j_{1}^{\prime}$ are even): if $w=w^{\prime}$ we would have $u \cdot O=v \cdot O$, but this is impossible since, by $(\star)$, the point $u \cdot O$ belongs to $\mathcal{C}\left(\sigma_{A C}^{+}, \frac{\pi}{3}\right)$ while $v \cdot O$ belongs to $a \cdot \mathcal{C}\left(\sigma_{A C}^{+}, \frac{\pi}{3}\right)$, and $\mathcal{C}\left(\sigma_{A C}^{+}, \frac{\pi}{3}\right) \cap a \cdot \mathcal{C}\left(\sigma_{A C}^{+}, \frac{\pi}{3}\right)=\emptyset$.

4.2. Non-geometric 3-manifolds. In this section we will prove the finiteness results Theorem 7 and Corollary 8 for non-geometric Riemannian 3-manifolds with bounded entropy and diameter. Recall that $\mathcal{M}_{\mathrm{ng}}^{\partial}(E, D)$ denotes the class of compact, orientable non-geometric 3-manifolds (with possibly empty, nonspherical boundary), possibly with torsion, endowed with Riemannian metrics with entropy and diameter bounded from above by two positive constants $E$ and $D$. We will first show the finiteness of fundamental groups of manifolds in $\mathcal{M}_{\mathrm{ng}}^{\partial}(E, D)$, and then explain how to deduce Corollary 8 from Theorem 7.

The proof of the finiteness of fundamental groups relies on the fact that the fundamental group of every non-geometric 3-manifold, closed or compact with non-spherical boundary components, admits a 4-acylindrical splitting: this fact 
was proved by [67] (see also [17] for further details). The splitting is relative either to the decomposition of $\pi_{1}(X)$ as a free product given by prime decomposition, or to the decomposition of $\pi_{1}(X)$ as an amalgamated product over rank 2 , abelian subgroups provided by the JSJ-decomposition, for irreducible manifolds, cf. §4 of [19]. We can then apply the entropy-cardinality inequality to the classical triangular presentation of $\pi_{1}(X)$ given by the Lemma 4.1, and proceed as in the proof of Theorem 6 , case (a).

Proof of Theorem 7. For any $X \in \mathcal{N}_{\mathrm{ng}}^{\partial}(E, D)$ pick $x \in X$ and let $M=D+\epsilon$. Then, consider the set $S_{M}$ of $M$-short generators at $x$. As $\pi_{1}(X)$ has a 4-acylindrical splitting, it follows from from Theorem 1 that

$$
E \geq \operatorname{Ent}(X) \geq \frac{1}{M} \operatorname{Ent}\left(\pi_{1}(X), S_{M}\right) \geq \frac{1}{488 M} \log \left(\left|S_{M}\right|-1\right)
$$

and letting $\epsilon \rightarrow 0$ we obtain $\left|S_{M}\right| \leq 1+e^{488 E D}=N(E, D)$. Therefore, $X$ admits a triangular presentation on a generating set of cardinality at most $N=N(E, D)$. Since the number of possible triangular presentations that can be build with letters from some subset $S$ of an alphabet $\mathcal{A}$ of $N$ letters does not exceed $2^{N+N^{3}}$, this concludes the proof.

Now, the following statement is consequence of several results of 3-dimensional geometry and topology. Since it relies on facts which are now folklore (and are sometimes only sketched in literature), we will provide a full proof in Appendix $\mathrm{B}$, together with all the references and the 3-dimensional topology tools needed for it.

Theorem B.1. There exist only finitely many pairwise non-diffeomorphic, compact orientable 3-manifolds without spherical boundary components with given fundamental group $G$.

Corollary 8 then follows from the fact that the fundamental groups of Riemannian manifolds in the class $\mathcal{M}_{\mathrm{ng}}^{\partial}(E, D)$ belong to a finite collection.

4.3. Ramified coverings. We briefly recall the construction of a cyclic ramified covering of a hyperbolic manifold of dimension $n \geq 2$, according to Gromov and Thurston [27]. Let $Z_{0}$ be a two-sided hypersurface with boundary in some closed, orientable n-manifold $X_{0}$, and call $R_{0}=\partial Z_{0}$ the (possibly disconnected) smooth, nonempty boundary. Cut $X_{0}$ along $Z_{0}$, thus obtaining a topological, compact manifold $\dot{X}_{0}$ with boundary; the boundary is given by two copies $Z_{0}^{-}, Z_{0}^{+}$of $Z_{0}$, with $Z_{0}^{-} \cap Z_{0}^{+}=R_{0}$. Then, consider the topological manifold $\hat{X}_{k}$ obtained by taking $k$ copies $\dot{X}_{i}$ of $\dot{X}_{0}$, and gluing $\dot{X}_{i}$ to $\dot{X}_{i+1}$ by identifying the boundaries $Z_{i}^{+}, Z_{i+1}^{-}$, for $i=1, \ldots, k-1$; finally, let $X_{k}$ be the closed manifold obtained by identifying $Z_{k}^{+}$to $Z_{1}^{-}$, and call $Z_{i}$ the boundaries so identified inside $X_{k}$. The 
resulting manifold $X_{k}$ can be given a smooth structure with a smooth projection onto the initial manifold $p: X_{k} \rightarrow X_{0}$ which is a smooth $k$-sheeted covering outside the ramification locus $R=p^{-1}\left(R_{0}\right)$; the ramification locus $R$ is the boundary of each $Z_{i}$, and around $R$ the projection writes as $(x, z) \mapsto\left(x, z^{k}\right)$, with respect to suitable coordinates for $X_{k}$ and for $X_{0}$, identifying the (trivial) normal bundles of $R$ and $R_{0}$ to $R_{0} \times D^{2}$.

Moreover, choosing $X_{0}$ hyperbolic and the submanifolds $Z_{0}, R_{0}$ totally geodesic in $X_{0}$, the new manifold $X_{k}$ can be given a singular, locally CAT(-1)-metric $g_{k}$ which makes of $\bigcup_{i} Z_{i}$ a (singular) totally geodesic hypersurface of $X_{k}$, with totally geodesic boundary $R$, and such that the restriction $\left.p\right|_{X_{k}-R}$ is a Riemannian covering (cf. [27]). Namely, $\bigcup_{i} Z_{i}$ looks like a $k$-paged book, consisting of $k$ copies of $Z$ joined together at $R$, each pair of consecutive pages forming an angle $2 \pi$ and a locally convex subset of $X_{k}$. The singular metric, around the ramification submanifold $R$, can be written as

$$
\cosh ^{2}(r) g_{0}+k \sinh ^{2}(r) d \theta^{2}+d r^{2},
$$

where $r$ represents the distance to $R$, and $g_{0}$ the metric of $Z_{0}$ (and where the first term does not appear when $n=2$ ). As shown in [27], this metric can then be smoothed to obtain a true Riemannian metric $g_{k}^{\epsilon}$ of strictly negative curvature $K\left(X_{k}\right) \leq-1$, and even pinched around -1 , provided that the normal injectivity radius of $R$ is sufficiently large. We will call a Riemannian manifold obtained by choosing any Riemannian metric on such $X_{k}$ (possibly with variable sectional curvature, of any possible sign, and not necessarily locally isometric to the base hyperbolic manifold $X_{0} \backslash R_{0}$ ), for any $k \geq 2$, a Riemannian ramified covering of $X_{0}$.

Proof of Corollary 9. Let $\widehat{X}_{k-1} \subset X_{k}$ be the glueing of the $\dot{X}_{i}$ 's, for $2 \leq i \leq k$. By Van Kampen theorem, $G_{k}=\pi_{1}\left(X_{k}\right)$ can be written as the amalgamated product $G_{1} *_{H} G_{k-1}$ of $G_{1}=\pi_{1}\left(\dot{X}_{1}\right)$ and $G_{k-1}=\pi_{1}\left(\hat{X}_{k-1}\right)$ along $H=$ $\pi_{1}\left(Z_{1}^{-} \cup Z_{1}^{+}\right)$, which is immersed in $G_{k-1}$ via the isomorphisms induced by the identifications $Z_{1}^{+} \simeq Z_{2}^{-}$and $Z_{1}^{-} \simeq Z_{k}^{+}$. Recall that the subset $Z:=$ $Z_{1} \cup Z_{k}$ of $X_{k}$ obtained by these identifications is a locally convex subset with respect to the singular, CAT(-1)-metric of $X_{k}$ described above, as well as the subsets $\dot{X}_{1}$ and $\widehat{X}_{k-1}$. The subgroup $H$ is then a malnormal subgroup of $G_{k}$ by Proposition C.1 of Appendix C.

Let us now show that the splitting $G_{k}=G_{1} *_{H} G_{k-1}$ is nontrivial. Call $p: \widetilde{X}_{k} \rightarrow X_{0}$ the universal covering map, let $\widetilde{Z}$ be a connected component of $p^{-1}(Z)$, let $\widetilde{X}_{1}$ and $\tilde{X}_{k-1}$ be the connected components respectively of $p^{-1}\left(\dot{X}_{1}\right)$ and $p^{-1}\left(\hat{X}_{k-1}\right)$ containing $\tilde{Z}$, and pick $\tilde{z}_{0} \in \widetilde{Z}$ projecting to $z_{0}=p\left(\tilde{z}_{0}\right)$. Notice that $\widetilde{Z}, \tilde{X}_{1}$ and $\tilde{X}_{k-1}$ are universal coverings of $Z, \dot{X}_{1}$ and $\hat{X}_{k-1}$ respectively, and that they are all convex subsets of $\tilde{X}_{k}$, since $Z, \dot{X}_{1}$ and $\hat{X}_{k-1}$ are locally convex (Steps 2 and 3 of Proposition C.1). Moreover, $H, G_{1}$ and $G_{k-1}$ act cocompactly 
on $\tilde{Z}, \tilde{X}_{1}$ and $\tilde{X}_{k-1}$ respectively. Then, if $H=G_{1}$ we would deduce that $\tilde{X}_{1}(\infty)=\widetilde{Z}(\infty)=L H$, hence that $\widetilde{X}_{1}=\widetilde{Z}$ (for given any $\tilde{x} \in \widetilde{X}_{1} \backslash \widetilde{Z}$, let $\tilde{\gamma}$ be the ray which extends $\left[\tilde{z}_{0}, \tilde{x}\right]$ beyond $\tilde{x}$ : this ray does not meet again $\widetilde{Z}$ or, by convexity, $\tilde{x}$ would belong to $\widetilde{Z}$; hence, it goes to infinity to some $\zeta \in \widetilde{Z}(\infty)$ and then, again, $\tilde{x}$ would belong to $\widetilde{Z}$ by convexity). This is a contradiction, by construction, as $Z \cap \dot{X}_{1}$ is a submanifold of $\dot{X}_{1}$. Therefore $H$ is strictly smaller than $G_{1}$; one analogously proves that $H \lesseqgtr G_{k-1}$.

Moreover, as $H$ is malnormal, then $[G: H]>2$, so one has $\left[G_{1}: H\right]>2$ and $\left[G_{k-1}: H\right]>2$ and the splitting is non-elementary. We conclude, by the discussion after Lemma 1.4, that $G$ is a 0 -step malnormal amalgamated product, i.e. it admits a 1-acylindrical splitting. We can then apply the entropy-cardinality inequality to a triangular presentation of $\pi_{1}(X)$, as in the proof of Theorem 6 and 7.

Namely, we choose some point $x \in X$ for every $X \in \mathcal{R}(E, D)$, we consider a triangular presentation of $\pi_{1}(X)$ by the $M$-short generating sets $S_{M}$, for $M=$ $D+\epsilon$, and as $d(x, g \cdot x) \leq M \cdot|g|_{S_{M}}$ for all $g \in S_{M}$, we deduce by Theorem 1 that $\left|S_{M}\right| \leq N(E, D)$. Therefore, the number of possible fundamental groups in $\mathcal{R}(E, D)$ is bounded in terms of $E, D$. Since every $X_{k}$ is an aspherical manifold admitting a CAT $(-1)$ metric, we infer the finiteness of homotopy types (and of diffeomorphisms types in dimension different from 4) as explained in the proof of Corollary 5.

\subsection{Higher dimensional graphs and cusp decomposable manifolds}

Proof of Corollary 10. By [25], Proposition 6.4, we know that the fundamental groups of irreducible higher graph manifolds admit 2-acylindrical splittings. On the other hand, the fundamental groups of cusp decomposable manifolds possess non-elementary, 1-acylindrical splittings. Actually, the decomposition of $\pi_{1}(X)$ corresponding to the cusp decomposition is obtained by identifying the cusp subgroups, and these subgroups are malnormal in the fundamental group of each bounded cusp manifold with horoboundary they belong to, and conjugately separated if they belong to the same group (cf. [23], or just apply Proposition C.1 of Appendix $\mathrm{C}$ to the whole, convex, cusp neighbourhoods). As a consequence, by Lemmas 1.6 and 1.7, the fundamental group of a cusp decomposable manifold can be presented as a 0 -step malnormal amalgamated product or $\mathrm{HNN}$-extension, and admits a 1-acylindrical splitting. Moreover, the Bass-Serre tree of the splittings corresponding to the decompositions of non-elementary higher graph or cusp decomposable manifolds is, by definition, neither a vertex nor a line. Therefore, the number of fundamental groups of manifolds in the classes $\mathcal{G}^{\partial}(E, D)$ and $\mathcal{C}(E, D)$ is finite, by the same argument used for Theorems 6 and 7. Since higher graph and cusp decomposable manifolds are aspherical (cf. [25], Corollary 3.3, and [49]) we immediately infer the finiteness of the homotopy types in $\mathcal{G}^{\partial}(E, D)$ and $\mathcal{C}(E, D)$. By the topological and differential rigidity properties of higher 
graph and cusp decomposable manifolds recalled in the introduction, we also deduce the finiteness of diffeomorphism types in $\mathcal{G}(E, D)$ and $\mathcal{C}(E, D)$.

Remark 4.3. The finiteness result holds, more generally, also for the diffeomorphism types of non-irreducible high dimensional graph manifold with boundary, admitting at least one internal walls with transverse fibers, and whose boundary components do not belong to surface pieces (see [25], §5).

\section{Appendices}

\section{A. Acylindrical splittings of hyperbolic 2-orbifolds}

The aim of this section is to give a self-contained proof of the following fact (which is a particular case of more general splitting results proved in [30]):

Proposition A.1. Let $\mathcal{O}$ be a compact 2-orbifold of hyperbolic type with conical singularities. If $\mathcal{O}$ is not a hyperbolic triangular orbifold, then $\pi_{1}^{\text {orb }}(\mathcal{O})$ admits a 2-acylindrical splitting.

Proof. First notice that all compact 2-orbifolds of hyperbolic type with non-empty boundary have orbifold fundamental group which is a non-trivial free product of finite and infinite cyclic groups, hence $\pi_{1}^{\text {orb }}(\mathcal{O})$ has a 0 -acylindrical, splitting in this case. Moreover, the splitting is necessarily non-elementary (otherwise the orbifold would be a disc with two singular points of order two, and it would not have negative orbifold Euler characteristic).

Assuming then that $\mathcal{O}$ is a compact, 2-orbifold of genus $g$ of hyperbolic type without boundary, which is not a hyperbolic triangular orbifold. By the formula for the orbifold Euler characteristic one of the following holds:

- $g>1$;

- $g=1$ and $\mathcal{O}$ has at least one singular point;

- $g=0$ and $\mathcal{O}$ has $m \geq 4$ singular points, at least one of which has order greater than 2;

- $g=-1$ and $\mathcal{O}$ has 2 singular points, one of which of order greater than 2;

- $g=-1$ and $\mathcal{O}$ has $m \geq 3$ singular points;

- $g=-2$ and $\mathcal{O}$ has at least one singular point;

- $g<-2$.

The proof then is obtained by cutting any such orbifold $\mathcal{O}$ into two 2-orbifolds with boundary, and using repeatedly the following 
Lemma A.2. If $\mathcal{O}=\mathcal{O}\left(g, h ; p_{1}, \ldots, p_{k}\right)$ is a compact 2-orbifold with boundary of hyperbolic type, the infinite cyclic subgroups $\left\langle d_{i}\right\rangle$, corresponding to the boundary curves form a collection of malnormal, conjugately separated subgroups of $\pi_{1}^{\text {orb }}(\mathcal{O})$.

The lemma can be checked directly by looking at the aforementioned presentations of the orbifold fundamental group: it is sufficient to notice that the boundary curves are represented by primitive elements of infinite order in a non-trivial free product of cyclic groups, different from $\mathbb{Z}_{2} * \mathbb{Z}_{2}$, which do not belong to the same conjugacy class. A more geometric justification to malnormality is that $\mathcal{O}$ can be given a geometric structure of a hyperbolic 2-orbifold with cusps, with the boundary subgroups $\left\langle d_{i}\right\rangle$ becoming the parabolic subgroups associated to the cusps.

Now, If $g \geq 1$, choose a simple closed curve $\delta$ which does not disconnect $|\mathcal{O}|$; after possibly modify the curve $\delta$ in order to avoid the singular points, cut $|\mathcal{O}|$ along that curve. We obtain a new orbifold $\mathcal{O}^{\prime}$ with genus $g-1$ and two new boundary components $\delta_{1}, \delta_{2}$; clearly, $\chi_{\text {orb }}\left(\mathcal{O}^{\prime}\right)=\chi_{\text {orb }}(\mathcal{O})<0$. Since $\mathcal{O}^{\prime}$ is an orbifold of hyperbolic type, the classes $d_{1}, d_{2}$, represented by $\delta_{1}$ and $\delta_{2}$ in the fundamental group of $\mathcal{O}^{\prime}$, generate two conjugately separated, malnormal subgroups in $\pi_{1}^{\text {orb }}\left(\mathcal{O}^{\prime}\right)$, by Lemma A.1. Then, by Lemma 1.7 we know that $\pi_{1}^{\text {orb }}(\mathcal{O})$ is the HNN-extension $\pi_{1}^{\text {orb }}\left(\mathcal{O}^{\prime}\right) *_{\varphi}$ defined by the isomorphism $\varphi:\left\langle d_{1}\right\rangle \rightarrow\left\langle d_{2}\right\rangle$, $\varphi\left(d_{1}\right)=d_{2}$; this yields a 2-acylindrical splitting of $\pi_{1}^{\text {orb }}(\mathcal{O})$. By construction, the Bass-Serre tree of this splitting is neither a point nor a line, so the splitting is non-elementary.

Assume now that $g=0$ and that $\mathcal{O}$ has at least $m \geq 4$ singular points, one of which of order $r \geq 3$. Consider a simple closed curve $\delta$ which separates $|\mathcal{O}|$ into two disc orbifolds $\mathcal{O}_{1}, \mathcal{O}_{2}$, each containing at least 2 singular points, with, let's say, $\mathcal{O}_{1}$ containing the singular point of order $r \geq 3$. Denoting by $d$ the classes represented by the boundary curve in each $\mathcal{O}_{i}$, the orbifold fundamental groups have presentations:

$$
\begin{aligned}
& \pi_{1}^{\mathrm{orb}}\left(\mathcal{O}_{1}\right)=\left\langle c_{1}, \ldots, c_{k}, d \mid c_{1} \cdots c_{k} d=1, c_{1}^{p_{1}}=\cdots=c_{k}^{p_{k}}=1\right\rangle, \\
& \pi_{1}^{\mathrm{orb}}\left(\mathcal{O}_{2}\right)=\left\langle c_{k+1}, \ldots, c_{m}, d \mid c_{k+1} \cdots c_{m} d=1, c_{k+1}^{p_{k+1}}=\cdots=c_{m}^{p_{m}}=1\right\rangle .
\end{aligned}
$$

Notice that $\chi\left(\mathcal{O}_{1}\right)=\chi\left(\left|\mathcal{O}_{1}\right|\right)-1-\sum_{i=1}^{k}\left(1-\frac{1}{p_{k}}\right)<0$, therefore the infinite cyclic subgroup $\langle d\rangle$ is a malnormal subgroup of $\pi_{1}^{\text {orb }}\left(\mathcal{O}_{1}\right)$, by Lemma A.1. Moreover, $\pi_{1}^{\text {orb }}(\mathcal{O})$ splits non-trivially as $\pi_{1}^{\text {orb }}\left(\mathcal{O}_{1}\right) *\langle d\rangle \pi_{1}^{\text {orb }}\left(\mathcal{O}_{2}\right)$, and Lemma 1.6 implies that this is a non-elementary, 2-acylindrical splitting.

If $g=-1$, consider a closed loop $\delta$ enclosing the singular points $x_{1}, \ldots, x_{m}$ and cut $\mathcal{O}$ along this loop. We obtain two orbifolds with boundary: a disc with $m$ singular points $\mathcal{O}_{1}$, and a Möbius strip $\mathcal{O}_{2}$. Observe that $\chi\left(\mathcal{O}_{1}\right)=\chi(\mathcal{O})<0$ and, calling again $d$ the classes represented by the boundary loops $\delta$ in each $\pi_{1}^{\text {orb }}\left(\mathcal{O}_{i}\right)$, the subgroup $\langle d\rangle$ is malnormal in $\pi_{1}^{\text {orb }}\left(\mathcal{O}_{1}\right)$ by Lemma A.2, while $\langle d\rangle$ 
is a subgroup of index two in $\pi_{1}^{\text {orb }}\left(\mathcal{O}_{2}\right)=\left\langle a, d \mid a^{2} d=1\right\rangle$. As $\pi_{1}^{\text {orb }}(\mathcal{O})=$ $\pi_{1}^{\text {orb }}\left(\mathcal{O}_{1}\right) *\langle d\rangle \pi_{1}^{\text {orb }}\left(\mathcal{O}_{2}\right)$, we have again by Lemma 1.6 that $\pi_{1}^{\text {orb }}(\mathcal{O})$ is a nontrivial, 1-step malnormal amalgamated product, and possesses a non-elementary, 2-acylindrical splitting.

Finally, if $g \leq-2$ then $\mathcal{O}$ can be cut along a boundary loop $\delta$ in two orbifolds $\mathcal{O}_{i}$, and we can assume that either $\mathcal{O}_{1}$ has genus 1 and at least one singular point, or $\mathcal{O}_{1}$ has genus greater than 1 and no singular points. In the first case, one has $\pi_{1}^{\text {orb }}\left(\mathcal{O}_{1}\right)=\left\langle a, c, d \mid a_{1}^{2} c d=c^{p}=1\right\rangle \cong \mathbb{Z} * \mathbb{Z}_{p}$, whereas in the second one $\pi_{1}^{\text {orb }}\left(\mathcal{O}_{1}\right)=\left\langle a_{1}, a_{2}, d\right\rangle \cong \mathbb{Z} * \mathbb{Z}$. In both cases, $\pi_{1}^{\text {orb }}(\mathcal{O})$ splits as a non-trivial amalgamated product $\pi_{1}^{\text {orb }}\left(\mathcal{O}_{1}\right) *\langle d\rangle \pi_{1}^{\text {orb }}\left(\mathcal{O}_{2}\right)$ with $\langle d\rangle$ malnormal in $\pi_{1}^{\text {orb }}\left(\mathcal{O}_{1}\right)$, which gives again a non-elementary 2 -acylindrical splitting.

\section{B. 3-manifolds with prescribed fundamental group}

The following statement is consequence of a number of classical results, which we will recall hereafter for the convenience of the reader:

Theorem B.1. There exist only finitely many pairwise non-diffeomorphic, compact orientable 3-manifolds without spherical boundary components with given fundamental group $G$.

To begin with, recall that in dimension 3 the homeomorphism type determines the diffeomorphism type, by the celebrated works of Moise, Munkres, and Whitehead [46], [48], and [68].

Now, Theorem B.1 is well known for closed 3-manifolds. Actually, if $X$ and $X^{\prime}$ are prime, closed, orientable, 3-manifolds with isomorphic fundamental groups, then $X$ and $X^{\prime}$ are homeomorphic, unless $X$ and $X^{\prime}$ are lens spaces; this follows from basic facts of 3-dimensional topology and from the solution of the Geometrization Conjecture (see, for instance, [2], Chapters 1 and 2). Moreover, by the classification of lens spaces, for every fixed $p \in \mathbb{N}$ there exists only a finite number of lens spaces $L(p, q)$ having $\mathbb{Z}_{p}$ as fundamental group (see for example [2], pp. 27-28). On the other hand, for non-prime, closed 3-manifolds, the statement follows by Kneser's theorem and the fact that the homeomorphism type of a connected sum is determined by the prime factors up to a finite number of choices, the indeterminacy being given by the orientations of the summands.

The proof of Theorem B.1 for general compact, orientable 3-manifolds with boundary is more tricky and due to Johannson ([31], Corollary 29.3) in the case of irreducible manifolds with incompressible boundary. ${ }^{7}$ Recall that a compact 3-manifold $X$ is irreducible if any embedded 2-sphere bounds a 3-ball. The same result was proved, independently, by Swarup ([63], Theorem A), without

${ }^{7}$ Johannson's statement is more general and requires the manifolds to be Haken. 
the incompressibility assumptions. However, the part of Swarup's proof dealing with possibly compressible boundary components invokes a proposition from [32] (namely, Proposition 3.9) that we were not able to track; since we noticed that this result, in more recent references like [2], is stated only for irreducible compact 3-manifolds with incompressible boundary, we find worth filling the details of the proof for general, compact manifolds with boundary without spherical boundary components, assuming Johannson's statement. We will closely follow Swarup's ideas, so no claim of originality is made.

Let us recall some basic terminology about 3-manifolds $X$ with boundary. A closed, properly embedded 2-disk $D \subset X$ (that is, such that $\partial D \subset \partial X$ ) is called essential if the loop $\partial D$ does not bound any embedded disk in $\partial X$. Two such disks $D, D^{\prime}$ are said to be parallel if there is an ambient isotopy sending $D$ into $D^{\prime}$. One says that $X$ has incompressible boundary if there are no essential disks.

\section{The surgery procedure for irreducible manifolds with compressible bound-} ary. Let $X$ be a compact, irreducible 3-manifold: a disk system for $X$ is a collection $\mathcal{C}$ of pairwise disjoint and non-parallel essential disks; the system is maximal if any collection $\mathcal{C}^{\prime}$ of essential disks properly containing $\mathcal{C}$ contains a pair of parallel disks. Assume that $X$ has compressible boundary: we can then choose a non-empty, maximal disk system $\mathcal{C}=\left\{D_{1}, \ldots, D_{r}\right\}$ and remove these disks from $X$. This procedure chops our irreducible manifold $X$ into a finite collection $\Gamma(X, \mathcal{C})$ of irreducible 3-manifolds with incompressible boundary $X_{1}, \ldots, X_{n}$ and finitely many 3-dimensional balls $B_{1}, \ldots, B_{m}$. Moreover, the collection $\Gamma(X, \mathcal{C})=\left\{X_{1}, \ldots, X_{n}, B_{1}, \ldots, B_{m}\right\}$ can be given a graph structure: the edges $d_{i}$ of $\Gamma(X, \mathcal{C})$ are in bijection with the disks $D_{i}$ of the maximal disk system $\mathcal{C}$, and two vertices $v, v^{\prime}$ of $\Gamma(X, \mathcal{C})$ (possibly with $v=v^{\prime}$ ) are connected by $d_{i}$ if the disk $D_{i}$ bounds the corresponding manifolds or balls.

The irreducible components $X_{1}, \ldots, X_{n}$ with incompressible boundary are uniquely determined up to diffeomorphism and do not depend on the particular maximal disc system $\mathcal{C}$ (see [44] pp. 167-168, or [43]); on the other hand, the number $k$ of balls arising from the surgery procedure may depend on the choice of $\mathcal{C}$. This procedure can be inverted: we can reconstruct the manifold $X$ from $\Gamma(X, \mathcal{C})$ by gluing back a 1 -handle, i.e. a copy of $D^{2} \times[0,1]$, for every edge of the graph: roughly speaking, $X$ appears as a "solid graph" whose vertices are the manifolds in the collection $\left\{X_{1}, \ldots, X_{n}, B_{1}, \ldots, B_{m}\right\}$ and whose edges are 1-handles connecting two (possibly equal) boundary components of the vertices. Using Van Kampen's theorem we see that the fundamental group of $X$ is isomorphic to a free product $\pi_{1}(X) \cong \pi_{1}\left(X_{1}\right) * \cdots * \pi_{1}\left(X_{n}\right) * \mathbb{F}_{k}$ where $k$ is the number (possibly zero) of cycles in the graph $\Gamma(X, \mathcal{C})$.

Clearly, the number $n$ of compact, irreducible 3-manifolds $X_{i}$ with incompressible boundary components obtained by the surgery procedure is bounded, 
by Grushko's theorem, by $N=n+k$. The next Lemma gives a bound of the numbers $r$ and $m$ of, respectively, 1-handles and balls appearing from the surgery procedure.

Lemma B.2. Let $N$ be the number of irreducible factors of $G=\pi_{1}(X)$ as a free product: then, $m \leq 2 N$ and $r \leq 3 N$.

Proof. We associate to $\Gamma(X, \mathcal{C})$ a graph of groups $\mathcal{G}(X, \mathcal{C})$, by assigning the group $G_{X_{i}}=\pi_{1}\left(X_{i}\right)$ to each vertex $X_{i}$, and the trivial groups to the vertices $B_{j}$ and to every edge $d_{i}$. Then, $\pi_{1}\left(\mathcal{G}_{Y}\right) \cong G_{X_{1}} * \cdots * G_{X_{n}} * \mathbb{F}_{k} \cong G$ exactly. Notice that, from the non-parallelism condition, the degree of the vertices of $\Gamma(X, \mathcal{C})$ corresponding to the 3-balls is at least 3 , unless the initial manifold was a solid torus, in which case the graph is just a loop and the collection of manifolds obtained after the surgery consists of a single 3-ball; therefore, we may assume that $\operatorname{deg}\left(B_{i}\right) \geq 3$ for $i=1, \ldots, m$. On the other hand, since the initial manifold has compressible boundary, we know as well that $\operatorname{deg}\left(X_{i}\right) \geq 1$ for each $i=1, \ldots, n$. Now, consider a maximal tree $\mathcal{T}$ in $\Gamma(X, \mathcal{C})$ : the maximal tree will have $n+m$ vertices and $n+m-1$ edges. Let $\mathrm{E}^{\prime}=\mathrm{E}(\Gamma(X, \mathcal{C})) \backslash \mathrm{E}(\mathcal{T})$. Observe that, by construction, the adjunction of each edge of $E^{\prime}$ to $\mathcal{T}$ corresponds to add a free factor isomorphic to an infinite cyclic group, so \#形 $=k$. Then,

$2 \cdot k+2 \cdot(n+m-1) \geq 2 \cdot \# \mathrm{E}(\Gamma(X, \mathcal{C}))=\sum_{i=1}^{n} \operatorname{deg}\left(X_{i}\right)+\sum_{i=1}^{m} \operatorname{deg}\left(B_{i}\right) \geq n+3 m$,

hence $m \leq(2 k+n-2)$ and $r=\# \mathrm{E}(\Gamma(X, \mathcal{C})) \leq k+n+m-1 \leq 3 k+2 n-3$ and we conclude that $m$ and $r$ are (roughly) bounded respectively by $2 N$ and $3 N$.

Proof of Theorem B.1 for orientable manifolds with boundary. Let $G$ be a (compact) 3-manifold group, whose decomposition as a free product has $N$ indecomposable factors. If $X$ is an orientable, compact manifold with boundary without spherical boundary components and fundamental group $G$, it has a prime decomposition as a connected sum of irreducible manifolds and copies of $S^{2} \times S^{1}$ (the only prime, non irreducible manifold without spherical boundary components), with at most $N$ factors. The homeomorphism type of a connected sum being uniquely determined by its factors and their orientations, it will then be enough to prove the theorem for irreducible manifolds. Now, by Lemma B.2, any compact, irreducible 3-manifold $X$ with fundamental group isomorphic to $G$ can be split using the surgery procedure in $n \leq N$ irreducible 3-manifolds $X_{i}$ with incompressible boundary and fundamental group $G_{i}$, plus a number $m \leq 2 N$ of 3-balls; and $X$ is obtained as a solid graph on these pieces, attaching at most $r \leq 3 N$ 1-handles. Moreover, notice that, by Kneser's Theorem (holding for 
irreducible 3-manifolds with incompressible boundary components), the fundamental group of each $X_{i}$ is indecomposable, hence isomorphic to one indecomposable factor of the free product decomposition of $G$. Now, by Johannson's theorem, for each indecomposable factor $G_{i}$ of $G$ there exist only finitely many nonhomeomorphic irreducible 3-manifolds $X_{i, \alpha}$ with incompressible boundary with fundamental group $G_{i}$. Moreover, any two disks $D, D^{\prime}$ in one of these $X_{i, \alpha}$ are isotopic, and there are only two isotopy classes of diffeomorphisms $D^{2} \rightarrow D^{2}$ (corresponding to the identity and to a reflection with respect to one axis); hence, once fixed two such pieces $X_{i, \alpha}$ and $X_{j, \beta}$, there are essentially two inequivalent ways of attaching a 1 -handle to them. Therefore, there are only finitely many manifolds which can be obtained as a solid graph on the (finitely many) pieces $X_{i, \alpha}$, which concludes the proof.

\section{Malnormal subgroups of CAT(0)-groups}

We start giving a method to detect malnormal subgroups in fundamental group of locally CAT( 0$)$-spaces.

Proposition C.1. Let $Z$ be a compact, locally convex subspace of a compact, complete locally $\mathrm{CAT}(0)$-space $X$. Assume that $X$ is negatively curved around $Z$ : then, $H=\pi_{1}(Z)$ is malnormal in $G=\pi_{1}(X)$.

By negatively curved around $Z$ we mean that $Z$ has a neighbourhood $U(Z)$ in $X$ such that $U(Z) \backslash Z$ is a locally CAT( $-k)$-space, for some $k>0$. Notice that this covers the case where $X$ is a complete Riemannian with sectional curvature $k_{X}<0$, with no a-priori negative upper bound on the curvature.

Proof. Let $\tilde{X} \rightarrow X$ the universal covering map: $\tilde{X}$ it is a CAT(0)-space. Let $\tilde{z}_{0} \in p^{-1}(Z) \subset \tilde{X}$ be a point projecting to $z_{0} \in Z$, and $H=\pi_{1}\left(Z, z_{0}\right)$. Finally, let $C_{\tilde{Z}} p^{-1}(Z)$ denote the connected component of $p^{-1}(Z)$ containing the point $\tilde{z}$, and $\widetilde{Z}_{\tilde{z}_{0}}$ the subset of $\tilde{X}$ obtained by lifting from $\tilde{z}_{0}$ any curve $\gamma$ of $Z$ based at $z_{0}$, and taking the endpoint $\tilde{\gamma}(1)$ of the lift $\tilde{\gamma}$.

1. $C_{\tilde{z}_{0}} p^{-1}(Z)=\widetilde{Z}_{\tilde{z}_{0}}$, and is a covering of $Z$. The fact that $C_{\tilde{z}} p^{-1}(Z)$ is a covering follows from ordinary theory of coverings, and the inclusion $\widetilde{Z}_{\tilde{z}_{0}} \subset$ $C_{\tilde{z}_{0}} p^{-1}(Z)$ is trivial. Conversely, if $\tilde{z} \in C_{\tilde{z}_{0}} p^{-1}(Z)$, it can be joined to $\tilde{z}_{0}$ by a curve $\tilde{\gamma}$ whose projection $\gamma$ stays in $Z$; hence $\tilde{z}=\tilde{\gamma}(1) \in \tilde{Z}_{\tilde{z}_{0}}$ by definition.

2. $\widetilde{Z}_{\tilde{z}_{0}}$ is the universal covering of $Z$, and $H$ injects in $G$. Actually, since locally CAT( 0$)$ spaces are locally convex, ${ }^{8}$ every class in $\pi_{1}\left(X, z_{0}\right)$ can be realized by a

\footnotetext{
${ }^{8} \mathrm{CAT}(0)$ spaces are assumed to be locally geodesic spaces (though non necessarily geodesic spaces), by definition.
} 
locally geodesic loop. Now, every locally geodesic loop $\gamma$ representing a class of $\pi_{1}\left(Z, z_{0}\right)$ lifts to a local geodesic $\tilde{\gamma}$ of $\tilde{X}$ from $\tilde{z}_{0}$ (the covering map being locally isometric). But every local geodesic in a CAT(0) space is a true geodesic, hence $\tilde{\gamma}$ is not closed: this shows that $\widetilde{Z}_{\tilde{z}_{0}}$ is simply connected, and that $\gamma$ does not represent the trivial element of $G$.

3. $\widetilde{Z}_{\tilde{z}_{0}}$, endowed with the length structure induced by $Z$, is isometrically embedded in $\tilde{X}$; therefore, it is a convex subset of $\tilde{X}$. In fact, since $Z$ is locally convex in $X$, the inclusion $\widetilde{Z}_{\tilde{z}_{0}} \subset \widetilde{X}$ is a local isometry; but, $\widetilde{X}$ being CAT(0), geodesics in $\tilde{X}$ are unique, which implies that $\widetilde{Z}_{\tilde{z}_{0}}$ is convex in $\tilde{X}$ and that the inclusion is a true isometric embedding.

4. $C_{g \tilde{z}_{0}} p^{-1}(Z)=g \cdot \widetilde{Z}_{\tilde{z}_{0}}$. As in (1) one sees that $C_{g \tilde{z}_{0}} p^{-1}(Z)=\widetilde{Z}_{g \tilde{z}_{0}}$ (the subset obtained by lifting from $g \tilde{z}_{0}$ any curve $\gamma$ of $Z$ with base point $z_{0}$ ), which clearly equals $g \cdot \widetilde{Z}_{g \tilde{z}_{0}}$.

5. $\operatorname{Stab}_{G}\left(\widetilde{Z}_{\tilde{z}_{0}}\right)=H$. The elements of $H$ clearly stabilize $\widetilde{Z}_{\tilde{z}_{0}}$ (recall that $h \in H$ acts on $\tilde{x} \in \tilde{X}$ by lifting from $\tilde{z}_{0}$ the composition of a geodesic $c$ from $z_{0}$ to $x=p(\tilde{x})$ with a loop $\gamma$ at $z_{0}$ representing $h$; so, the final point of the lift $\widetilde{\gamma c}$ belongs to $\widetilde{Z}_{\tilde{z}_{0}}$ by definition of $\left.\widetilde{Z}_{\tilde{z}_{0}}\right)$. Conversely: if $g \in \operatorname{Stab}_{G}\left(\widetilde{Z}_{\tilde{z}_{0}}\right)$, then $g \tilde{z}_{0} \in \widetilde{Z}_{\tilde{z}_{0}}$, and then the geodesic $\tilde{\gamma}$ joining $\tilde{z}_{0}$ to $g \tilde{z}_{0}$ stays in $\widetilde{Z}_{\tilde{z}_{0}}$ (since this is a convex subset of $\tilde{X}$ ). As $g$ is represented by the projection $\gamma$ of $\tilde{\gamma}$ in $X$, which is included in $Z$, then $g \in \pi_{1}(Z)=H$.

6. $\operatorname{Stab}_{G}\left(g \cdot \widetilde{Z}_{\tilde{z}_{0}}\right)=g H g^{-1}$, and the number of connected components of $p^{-1}(Z)$ is in bijection with the cosets space $G / H$. Both assertions follow from (4) and (5).

7. Every $h \in H$ acts on $\tilde{X}$ by hyperbolic isometries, and the subset $\operatorname{Min}(h)$ where the displacement function $d(\tilde{x}, h \tilde{x})$ attains its minimum is included in $\widetilde{Z}_{\tilde{z}_{0}}$. Since the action of $G=\pi_{1}\left(X, z_{0}\right)$ on $\tilde{X}$ is cocompact and without fixed points, then every element of $G$ acts on $\widetilde{X}$ by hyperbolic isometries. We shall now prove that $\operatorname{Min}(h)$ is entirely included in $\widetilde{Z}_{\tilde{z}_{0}}$, for every $h \in H$. Actually, let $\tilde{x}_{0}$ be an arbitrary point of minimum for the displacement function $s_{h}(\tilde{x})=d(\tilde{x}, h \tilde{x})$, and consider the projection $p: \tilde{X} \rightarrow \widetilde{Z}_{\tilde{z}_{0}}$ (this is well defined, since $\widetilde{Z}_{\tilde{z}_{0}}$ is a convex subset). As $\widetilde{Z}_{\tilde{z}_{0}}$ is invariant under $h$ by (5), and since $p$ is a projection, we have $h \cdot p\left(\tilde{x}_{0}\right)=p\left(h \cdot \tilde{x}_{0}\right)$. Therefore

$$
d\left(p\left(\tilde{x}_{0}\right), h \cdot p\left(\tilde{x}_{0}\right)\right)=d\left(p\left(\tilde{x}_{0}\right), p\left(h \cdot \tilde{x}_{0}\right)\right) \leq d\left(\tilde{x}_{0}, h \cdot \tilde{x}_{0}\right) .
$$

This shows that the point $p\left(\tilde{x}_{0}\right) \in \widetilde{Z}_{\tilde{z}_{0}}$ also realizes the minimum of $s_{h}(x)$. By the $h$-invariance and the convexity of $\operatorname{Min}(h)$, we deduce that the orbits $\left\{h^{n} \cdot \tilde{x}_{0}\right\}$ 
and $\left\{h^{n} \cdot \tilde{p}\left(x_{0}\right)\right\}$ lie on two parallel geodesics $\gamma$ and $p(\gamma)$, entirely included in $\operatorname{Min}(h)$; moreover, $p(\gamma) \subset \widetilde{Z}_{\tilde{z}_{0}}$, as $\widetilde{Z}_{\tilde{z}_{0}}$ is convex. So, $\operatorname{Min}(h)$ contains a flat band bounding $\gamma$ and $p(\gamma)$; the lifted neighbourhood $\widetilde{U}\left(\widetilde{Z}_{\tilde{z}_{0}}\right)$ of $\widetilde{Z}_{\tilde{z}_{0}}$ being strictly negative curved outside $\widetilde{Z}_{\tilde{z}_{0}}$, this shows that $\gamma$ and $\tilde{x}_{0}$ are necessarily included in $\widetilde{Z}_{\tilde{z}_{0}}$.

8. $\quad H$ is malnormal in $G$. Assume that there exists $h \in H^{*}$ and $g \in G$ such that $h^{\prime}=g h g^{-1} \in H$. By (7), $\operatorname{Min}(h)$ is included in $\widetilde{Z}_{\tilde{z}_{0}}=C_{\tilde{z}_{0}} p^{-1}(Z)$; but as $h^{\prime}=g h g^{-1}$ is in $H$, we also have $\operatorname{Min}\left(h^{\prime}\right) \subset C_{\tilde{z}_{0}} p^{-1}(Z)$. However, $\operatorname{Min}\left(h^{\prime}\right)=\operatorname{Min}\left(g h g^{-1}\right)=g \cdot \operatorname{Min}(h)$ is included in $g \cdot \widetilde{Z}_{\tilde{z}_{0}}=C_{g \tilde{z}_{0}} p^{-1}(Z)$, which is disjoint from $C_{\tilde{z}_{0}} p^{-1}(Z)$ if $g \notin H$, by (6). This shows that $g \in H$ and that $H$ is malnormal in $G$.

\section{References}

[1] A. Adem, J. Leida, and Y. Ruan, Orbifolds and stringy topology. Cambridge Tracts in Mathematics, 171. Cambridge University Press, Cambridge, 2007. Zbl 1157.57001 MR 2359514

[2] M. Aschenbrenner, S. Friedl, and H. Wilton, 3-manifolds groups. EMS Series of Lectures in Mathematics. European Mathematical Society (EMS), Zürich, 2015. Zbl 1326.57001 MR 3444187

[3] M. T. Anderson and J. Cheeger, $C^{\alpha}$-compactness for manifolds with Ricci curvature and injectivity radius bounded below. J. Differential Geom. 35 (1992), no. 2, 265-281. Zbl 0774.53021 MR 1158336

[4] C. S. Aravinda and F. T. Farrell, Twisted doubles and nonpositive curvature. Bull. Lond. Math. Soc. 41 (2009), no. 6, 1053-1059. Zbl 1185.53035 MR 2575336

[5] G. N. Arzhantseva and I. G. Lysenok, A lower bound on the growth of word hyperbolic groups. J. London Math. Soc. (2) 73 (2006), no. 1, 109-125. Zbl 1093.20024 MR 2197373

[6] W. Ballmann, Lectures on spaces of nonpositive curvature. With an appendix by M. Brin. DMV Seminar, 25. Birkhäuser Verlag, Basel, 1995. Zbl 0834.53003 MR 1377265

[7] A. Bartels and W. Lück, The Borel conjecture for hyperbolic and CAT(0)-groups. Ann. of Math. (2) 175 (2012), no. 2, 631-689. Zbl 1256.57021 MR 2993750

[8] G. Besson, G. Courtois, S. Gallot, and A. Sambusetti, Curvature-free Margulis lemma for Gromov-hyperbolic spaces. Preprint, 2017. arXiv:1712.08386

[9] M. Boileau, S. Maillot, and J. Porti, Three-dimensional orbifolds and their geometric structures. Panoramas et Synthèses, 15. Société Mathématique de France, Paris, 2003. Zbl 1058.57009 MR 2060653 
[10] F. Bonahon, Geometric structures on 3-manifolds. In R. J. Daverman and R. B. Sher (eds.), Handbook of geometric topology. North-Holland, Amsterdam, 2002, 93-164. Zbl 0997.57032 MR 1886669

[11] J. Borzellino, Orbifolds of maximal diameter. Indiana Univ. Math. J. 42 (1993), no. 1, 37-53. Zbl 0801.53031 MR 1218706

[12] E. Breuillard, B. Green, and T. Tao, The structure of approximate groups. Publ. Math. Inst. Hautes Études Sci. 116 (2012), 115-221. Zbl 1260.20062 MR 3090256

[13] M. R. Bridson and A. Haefliger, Metric spaces of non-positive curvature. Grundlehren der Mathematischen Wissenschaften, 319. Springer-Verlag, Berlin, 1999.

Zbl 0988.53001 MR 1744486

[14] M. Bucher and P. de la Harpe Free products with amalgamation and HNN-extension of uniformly exponential growth. Mat. Zametki 67 (2000), no. 6, 811-815. In Russian. English translation, Math. Notes 67 (2000), no. 5-6, 686-689. Zbl 0998.20025 MR 1820635

[15] P. Buser and H. Karcher, Gromov's almost flat manifolds. Astérisque 81. Société Mathématique de France, Paris, 1981. 148 pp. Zbl 0459.53031 MR 0619537

[16] M. Bucher and A. Talambutsa, Exponential growth rates of free and amalgamated products. Israel J. Math. 212 (2016), no. 2, 521-546. Zbl 1350.20020 MR 3505396

[17] F. Cerocchi, On the peripheral subgroups of irreducible 3-manifold groups and acylindrical splittings. Preprint, 2017. arXiv:1705.06124

[18] F. Cerocchi and A. Sambusetti, Quantitative bounded distance theorem and Margulis' lemma for $\mathbb{Z}^{n}$-actions, with applications to homology. Groups Geom. Dyn. 10 (2016), no. 4, 1227-1247. Zbl 1365.53044 MR 3605032

[19] F. Cerocchi and A. Sambusetti, Local topological rigidity of non-geometric 3manifolds. Geom. Topol. 23 (2019), no. 6, 2899-2927. Zbl 07142691 MR 4039182

[20] J. Cheeger, Finiteness theorems for Riemannian manifolds. Amer. J. Math. 92 (1970), 61-74. Zbl 0194.52902 MR 0263092

[21] J. Cheeger and T. H. Colding, Lower bounds on Ricci curvature and the almost rigidity of warped products. Ann. of Math. (2) 144 (1996), no. 1, 189-237. Zbl 0865.53037 MR 1405949

[22] P. de la Harpe, Topics in geometric group theory. Chicago Lectures in Mathematics. University of Chicago Press, Chicago, IL, 2000. Zbl 0965.20025 MR 1786869

[23] P. de la Harpe and C. Weber, Malnormal subgroups and Frobenius groups: basics and examples. Confluentes Math. 6 (2014), no. 1, 65-76. With an appendix by D. Osin. Zbl 1327.20030 MR 3266885

[24] C. Farsi, Orbifold spectral theory. Rocky Mountain J. Math. 31 (2001), no. 1, 215-235. Zbl 0977.58025 MR 1821378

[25] R. Frigerio, F. Lafont, and A. Sisto, Rigidity of high dimensional graph manifolds. Astérisque 372 (2015), xxI+177 pp. Zbl 1333.53001 MR 3444648

[26] K. Fukaya, Theory of convergence for Riemannian orbifolds. Japan. J. Math. (N.S.) 12 (1986), no. 1, 121-160. Zbl 0654.53044 MR 0914311 
[27] M. Gromov and W. Thurston, Pinching constants for hyperbolic manifolds. Invent. Math. 89 (1987), no. 1, 1-12. Zbl 0646.53037 MR 0892185

[28] K. Grove and P. Petersen, Bounding homotopy types by geometry. Ann. of Math. (2) 128 (1988), no. 1, 195-206. Zbl R0951512 MR 0655.53032

[29] K. Grove, P. Petersen, and J.-Y. Wu, Geometric finiteness theorems via controlled topology. Invent. Math. 99 (1990), no. 1, 205-213. Zbl 0747.53033 MR 1029396

[30] V. Guirardel and G. Levitt, JSJ decompositions of groups. Astérisque 395 (2017), viI+165 pp. Zbl 1391.20002 MR 3758992

[31] K. Johannson, Homotopy equivalences of 3-manifolds with boundaries. Lecture Notes in Mathematics, 761. Springer, Berlin, 1979. Zbl 0412.57007 MR 0551744

[32] K. Johannson, On exotic homotopy equivalences of 3-manifolds. In J. C. Cantrell (ed.), Geometric topology. (Athens, GA, August 1-12, 1977.) Academic Press, New York and London, 1979, 101-111. Zbl 0476.57003 MR 0537729

[33] M. W. Hirsch and B. Mazur, Smoothings of piecewise linear manifolds. Annals of Mathematics Studies, 80. Princeton University Press, Princeton, N.J., and University of Tokyo Press, Tokyo, 1974. Zbl 0298.57007 MR 0415630

[34] I. Kapovich and R. Weidmann, Acylindrical accessibility for groups acting on R-trees. Math. Z. 249 (2005), no. 4, 773-782. Zbl 1080.20035 MR 2126215

[35] V. Kapovitch and B. Wilking, Structure of fundamental group of manifolds with Ricci curvature bounded below. Preprint, 2011. arXiv:1105.5955

[36] A. Karrass and D. Solitar, The free product of two groups with a malnormal amalgamated subgroup. Canadian J. Math. 23 (1971), 933-959. Zbl 0247.20028 MR 0314992

[37] R. C. Kirby and L. C. Siebenmann, Foundational essays on topological manifolds, smoothings and triangulations. With notes by J. Milnor and M. Atiyah. Annals of Mathematics Studies, 88. Princeton University Press, Princeton, N.J., and University of Tokyo Press, Tokyo, 1977. Zbl 0361.57004 MR 0645390

[38] G. Knieper, Spherical means on compact Riemannian manifolds of negative curvature. Differential Geom. Appl. 4 (1994), no. 4, 361-390. Zbl 0812.53043 MR 1306567

[39] T. Lance, Differentiable structures on manifolds. In S. Cappell, A. Ranicki, and J. Rosenberg (eds.), Surveys on surgery theory. Papers dedicated to C. T. C. Wall. Vol. 1. Annals of Mathematics Studies, 145. Princeton University Press, Princeton, N.J., 2000, 73-104. Zbl 0941.57027 MR 1747531

[40] B. Leeb, 3-manifolds with(out) metrics of non-positive curvature. Invent. Math 122 (1995), no. 2, 277-289. Zbl 0840.53031 MR 1358977

[41] R. C. Lyndon and P. E. Schupp, Combinatorial group theory. Ergebnisse der Mathematik und ihrer Grenzgebiete, 89. Springer-Verlag, Berlin etc., 1977. Zbl 0368.20023 MR 0577064

[42] A. Manning, Topological entropy for geodesic flows. Ann. of Math. (2) 110 (1979), no. 3, 567-573. Zbl 0426.58016 MR 0554385 
[43] B. Martelli, An introduction to geometric topology. Preprint, 2016. arXiv:1610.02592

[44] S. Matveev, Algorithmic topology and classification of 3-manifolds. Algorithms and Computation in Mathematics, 9. Springer-Verlag, Berlin, 2003. Zbl 1048.57001 MR 1997069

[45] A. Minasyan and D. Osin, Acylindrical hyperbolicity of groups acting on trees. Math. Ann. 362 (2015), no. 3-4, 1055-1105. Zbl 1360.20038 MR 3368093

[46] E. Moise, Affine structures in 3-manifolds. V. The triangulation theorem and Hauptvermutung. Ann. of Math. (2) 56 (1952), 96-114. Zbl 0048.17102 MR 0048805

[47] G. Moreno and M. E. Stypa, On the vertex-to-edge duality between the Cayley graph and the coset geometry of von Dyck groups. Math. Slovaca 66 (2016), no. 3, 527-538. Zbl 1389.05169 MR 3543715

[48] J. Munkres, Obstructions to the smoothing of piecewise-differentiable homeomorphisms. Ann. of Math. (2) 72 (1960), 521-554. Zbl 0108.18101 MR 0121804

[49] T. T. Nguyen Phan, Smooth (non)-rigidity of cusp-decomposable manifolds. Comment. Math. Helv. 87 (2012), no. 4, 789-804. Zbl 1269.53042 MR 2984571

[50] D. Osin, Acylindrically hyperbolic groups. Trans. Amer. Math. Soc. 368 (2016), no. 2, 851-888. Zbl 1380.20048 MR 3430352

[51] M. Peigné and A. Sambusetti, Entropy rigidity of negatively curved manifolds of finite volume. Math. Z. 293 (2019), no. 1-2, 609-627. Zbl 07106146 MR 4002292

[52] S. Peters, Cheeger's finiteness theorem for diffeomorphism classes of Riemannian manifolds. J. Reine Angew. Math. 349 (1984), 77-82. Zbl 0524.53025 MR 0743966

[53] E. Proctor, Orbifold homeomorphism finiteness based on geometric constraints. Ann. Global Anal. Geom. 41 (2012), no. 1, 47-59. Zbl 1236.53039 MR 2860396

[54] E. Proctor and E. Stanhope, Spectral and geometric bounds on 2-orbifolds diffeomorphism type. Differential Geom. Appl. 28 (2010), no. 1, 12-18. Zbl 1185.58017 MR 2579379

[55] G. Reviron, Espaces de longueur d'entropie majoreée : rigidité topologique, adhérence des variétés, noyau de la chaleur. Ph.D. thesis. 2005, Institut Fourier, Grenoble, 2005.

[56] G. Reviron, Rigidité sous l'hypothèse « entropie majorée » et applications. Comment. Math. Helv. 83 (2008), no. 4, 815-846. Zbl 1153.53027 MR 2442964

[57] I. Satake, On a generalization of the notion of manifold. Proc. Nat. Acad. Sci. U.S.A. 42 (1956), 359-363. Zbl 0074.18103 MR 0079769

[58] P. Scott, The geometries of 3-manifolds. Bull. London Math. Soc. 15 (1983), no. 5, 401-487. Zbl 0561.57001 MR 0705527

[59] Z. Sela, Acylindrical accessibility for groups. Invent. Math. 129 (1997), no. 3, 527-565. Zbl 0887.20017 MR 1465334

[60] J. P. Serre, Trees. Translated from the French by J. Stillwell. Springer-Verlag, Berlin etc., 1980. Zbl 0548.20018 MR 0607504

[61] A. Sisto, Contracting elements and random walks. J. Reine Angew. Math. 742 (2018), 79-114. Zbl 06930685 MR 3849623 
[62] E. Stanhope, Spectral bounds on orbifold isotropy. Ann. Global Anal. Geom. 27 (2005), no. 4, 355-375. Zbl 1085.58026 MR 2155380

[63] A. Swarup, Two finiteness properties in 3-manifolds. Bull. London Math. Soc. 12 (1980), no. 4, 296-302. Zbl 0457.57007 MR 0576979

[64] W. P. Thurston, Three dimensional manifolds, Kleinian groups and Hyperbolic geometry. Bull. Amer. Math. Soc. (N.S.) 6 (1982), no. 3, 357-381. Zbl 0496.57005 MR 0648524

[65] W. P. Thurston, The geometry and topology of 3-manifolds. Lecture notes, 1980. http://library.msri.org/books/gt3m/

[66] A. Weinstein, On the homotopy type of positively pinched manifolds. Arch. Math. (Basel) 18 (1967), 523-524. Zbl 0166.17601 MR 0220311

[67] H. Wilton and P. Zalesskii, Profinite properties of graph manifolds. Geom. Dedicata 147 (2010), 29-45. Zbl 1204.57019 MR 2660565

[68] J. H. C. Whitehead, Manifolds with transverse fields in euclidean space. Ann. of Math. (2) 73 (1961), 154-212. Zbl 0096.37802 MR 0124917

Received July 16, 2019

Filippo Cerocchi, Dipartimento di Matematica "G. Castelnuovo",

Università di Roma "La Sapienza”, P.le Aldo Moro 5, 00185 Roma, Italia

e-mail: fcerocchi@gmail.com

Andrea Sambusetti, Dipartimento di Matematica "G. Castelnuovo", Università di Roma "La Sapienza", P.le Aldo Moro 5, 00185 Roma, Italia

e-mail: sambuset@mat.uniroma1.it 